\title{
Non-uniqueness for the Transport Equation with Sobolev Vector Fields
}

\section{Stefano Modena ${ }^{1}$ - László Székelyhidi $\mathrm{Jr}^{1}{ }^{1}$}

Received: 25 April 2018 / Accepted: 2 December 2018 / Published online: 7 December 2018

(c) The Author(s) 2018

\section{Abstract}

We construct a large class of examples of non-uniqueness for the linear transport equation and the transport-diffusion equation with divergence-free vector fields in Sobolev spaces $W^{1, p}$.

Keywords Transport equation - Renormalized solutions · Convex integration · Non-uniqueness $\cdot h$-principle

\section{Introduction}

This paper concerns the problem of (non)uniqueness of solutions to the transport equation in the periodic setting

$$
\begin{aligned}
\partial_{t} \rho+u \cdot \nabla \rho & =0, \\
\rho_{\mid t=0} & =\rho^{0}
\end{aligned}
$$

where $\rho:[0, T] \times \mathbb{T}^{d} \rightarrow \mathbb{R}$ is a scalar density, $u:[0, T] \times \mathbb{T}^{d} \rightarrow \mathbb{R}^{d}$ is a given vector field and $\mathbb{T}^{d}=\mathbb{R}^{d} / \mathbb{Z}^{d}$ is the $d$-dimensional flat torus.

Unless otherwise specified, we assume in the following that $u \in L^{1}$ is incompressible, i.e.

$$
\operatorname{div} u=0
$$

in the sense of distributions. Under this condition, (1) is formally equivalent to the continuity equation

$$
\partial_{t} \rho+\operatorname{div}(\rho u)=0 .
$$

\footnotetext{
László Székelyhidi Jr.

laszlo.szekelyhidi@math.uni-leipzig.de

Stefano Modena

stefano.modena@math.uni-leipzig.de

1 Institut für Mathematik, Universität Leipzig, 04109 Leipzig, Germany
} 
It is well known that the theory of classical solutions to (1)-(2) is closely connected to the ordinary differential equation

$$
\begin{aligned}
\partial_{t} X(t, x) & =u(t, X(t, x)), \\
X(0, x) & =x,
\end{aligned}
$$

via the formula $\rho(t, X(t, x))=\rho^{0}(x)$. In particular, for Lipschitz vector fields $u$ the well-posedness theory for (1)-(2) follows from the Cauchy-Lipschitz theory for ordinary differential equations applied to (5); on the other side, the inverse flow map $\Phi(t):=X(t)^{-1}$ solves the transport equation

$$
\begin{aligned}
& \partial_{t} \Phi+(u \cdot \nabla) \Phi=0, \\
& \left.\Phi\right|_{t=0}=\mathrm{id} .
\end{aligned}
$$

There are several PDE models, related, for instance, to fluid dynamics or to the theory of conservation laws (see for instance [19,25,33,35,36]), where one has to deal with vector fields which are not necessarily Lipschitz, but have lower regularity and therefore it is important to investigate the well-posedness of (1)-(2) in the case of non-smooth vector fields.

Starting with the groundbreaking work of DiPerna-Lions [26] there is a wealth of well-posedness results for vector fields which are Sobolev or BV (we refer to the recent survey [6], see also below) and in particular in recent years a lot of effort has been devoted to understanding how far the regularity assumptions can be relaxed. The main goal of this paper is to provide a lower bound on the regularity assumptions by showing, to our knowledge for the first time, that well-posedness can fail quite spectacularly even in the Sobolev setting, with $u \in C_{t} W_{x}^{1, \tilde{p}}:=C\left([0, T] ; W^{1, \tilde{p}}\left(\mathbb{T}^{d}\right)\right)$ (see Theorem 1.2 for the precise statement). The mechanism we exploit to produce such "failure of uniqueness" is so strong that it can be applied also to the transportdiffusion equation

$$
\partial_{t} \rho+\operatorname{div}(\rho u)=\Delta \rho
$$

thus producing Sobolev vector fields $u \in C_{t} W_{x}^{1, \tilde{p}}$ for which uniqueness of solutions to (7)-(2) fails in the class of densities $\rho \in C_{t} L^{p}$ (see Theorem 1.9).

Both theorems can be generalized as follows: we can construct vector fields with arbitrary large regularity $u \in W^{\tilde{m}, \tilde{p}}, \tilde{m} \in \mathbb{N}$, for which uniqueness of solutions to (1)(2) or (7)-(2) fails, in the class of densities $\rho \in W^{m, p}$, with arbitrary large $m \in \mathbb{N}$; moreover, we can do that even when on the r.h.s. of (7) there is a higher order diffusion operator (see Theorems 1.6 and 1.10).

Before stating the precise statements of these results, we present a brief (and far from complete) overview of the main well-posedness achievements present in the literature. We start with the analysis of the well-posedness for the transport equation in class of bounded densities, then we pass to the analysis of well-posedness for the transport equation in the class of $L^{p}$-integrable densities, with the statement of our Theorems 1.2 and 1.6 and finally we discuss the transport-diffusion equation, with the statements of our Theorems 1.9 and 1.10. The last part of this introduction is devoted to a brief overview of the main techniques used in our proofs. 


\subsection{The Case of Bounded Densities}

The literature about rough vector fields mainly concerns the well-posedness of (1)-(2) in the class of bounded densities, $\rho \in L^{\infty}$. The reason for that can be found in the fact that the scientific community has been mainly interested in the well-posedness of ODE (5) and has used the PDE as a tool to attack the ODE problem: the general strategy is that a well-posedness result for the transport equation in the class of bounded densities yields a unique solution to the PDE (6) and thus one tries to prove that the flow $X(t):=\Phi(t)^{-1}$ is the unique meaningful solution, in the sense of regular Lagrangian flow, to the ODE (5). We refer to [6] for a precise definition of the notion of regular Lagrangian flow and for a detailed discussion about the link between the Eulerian and the Lagrangian approach.

Let us observe that for $\rho \in L^{\infty}$ the quantity $\rho u \in L^{1}$ and thus one can consider solutions to (1) (or, equivalently, to (4), since we are assuming incompressibility of the vector field) in distributional sense: $\rho$ is a distributional or weak solution if

$$
\int_{0}^{T} \int_{\mathbb{T}^{d}} \rho\left[\partial_{t} \varphi+u \cdot \nabla \varphi\right] d x d t=0
$$

for every $\varphi \in C_{c}^{\infty}\left((0, T) \times \mathbb{T}^{d}\right)$. It is usually not difficult to prove existence of weak solutions, even if the vector field is very rough, taking advantage of the linearity of the equation. A much bigger issue is the uniqueness problem.

The first result in this direction dates back to DiPerna and Lions [26], when they proved uniqueness, in the class of bounded densities, for vector fields $u \in L_{t}^{1} W_{x}^{1,1}$ with bounded divergence. This result was extended in 2004 by Ambrosio [5] to vector fields $u \in L_{t}^{1} B V_{x} \cap L^{\infty}$ and with bounded divergence (see also [16,17]) and very recently by Bianchini and Bonicatto [8] for vector fields $u \in L_{t}^{1} B V_{x}$ which are merely nearly incompressible (see, for instance, [6] for a definition of nearly incompressibility).

The proofs of these results are very subtle and involves several deep ideas and sophisticated techniques. We could however try to summarize the heuristic behind all of them as follows: (very) roughly speaking, a Sobolev or BV vector field $u$ is Lipschitz-like (i.e. $D u$ is bounded) on a large set and there is just a small "bad" set, where $D u$ is very large. On the big set where $u$ is "Lipschitz-like", the classical uniqueness theory applies. Non-uniqueness phenomena could thus occur only on the small "bad" set. Uniqueness of solutions in the class of bounded densities is then a consequence of the fact that a bounded density $\rho$ can not "see" this bad set, or, in other words, cannot concentrate on this bad set.

With this rough heuristic in mind it is also perhaps not surprising that the theory cited above is heavily measure-theoretic. Nevertheless, the well-posedness for the ODE (5) fundamentally relies on the analysis and well-posedness theory of the associated PDE (1). More precisely in DiPerna and Lions [26], introduced the notion of renormalized solution. One calls a density $\rho \in L_{t x}^{1}$ renormalized for (1) (for given $u$ ), if for any $\beta \in L^{\infty}(\mathbb{R}) \cap C^{1}(\mathbb{R})$ it holds

$$
\partial_{t} \beta(\rho)+u \cdot \nabla \beta(\rho)=0
$$


in the sense of distributions. Analogously to entropy-conditions for hyperbolic conservation laws, (9) provides additional stability under weak convergence. Key to the well-posedness theory is then showing that any bounded distributional solution $\rho$ of (1) is renormalized - this is done by showing convergence of the commutator

$$
(u \cdot \nabla \rho)_{\varepsilon}-u_{\varepsilon} \cdot \nabla \rho_{\varepsilon} \rightarrow 0
$$

arising from suitable regularizations.

As we mentioned, uniqueness at the PDE level in the class of bounded densities implies, in all the cases considered above, uniqueness at the ODE level (again in the sense of the regular Lagrangian flow). On the other hand, based on a self-similar mixing example of Aizenmann [1], Depauw [24] constructed an example of nonuniqueness for weak solutions with $\rho \in L^{\infty}\left((0, T) \times \mathbb{T}^{d}\right)$ and $u \in L^{1}\left(\varepsilon, 1 ; B V\left(\mathbb{T}^{d}\right)\right)$ for any $\varepsilon>0$ but $u \notin L^{1}\left(0,1 ; B V\left(\mathbb{T}^{d}\right)\right)$. This example has been revisited in [3, $4,17,37]$. It should be observed, though, that the phenomenon of non-uniqueness in such "mixing" examples is Lagrangian in the sense that it is a consequence of the degeneration of the flow map $X(t, x)$ as $t \rightarrow 0$; in particular, once again, the link between (1) and (5) is crucial.

\subsection{The Case of Unbounded Densities}

There are important mathematical models, related, for instance, to the Boltzmann equation (see [25]), incompressible 2D Euler [19], or to the compressible Euler equations, in which the density under consideration is not bounded, but it belongs just to some $L_{t}^{\infty} L_{x}^{p}$ space. It is thus an important question to understand the well-posedness of the Cauchy problem (1)-(2) in such larger functional spaces.

As a first step, we observe that for a density $\rho \in L_{t}^{\infty} L_{x}^{p}$ and a field $u \in L_{t}^{1} L_{x}^{1}$, the product $\rho u$ is not well defined in $L^{1}$ and thus the notion of weak solution as in (8) has to be modified. There are several possibilities to overcome this issue. We mention two of them: either we require that $u \in L_{t}^{1} L_{x}^{p^{\prime}}$, where $p^{\prime}$ is the dual Hölder exponent to $p$, or we consider a notion of solution which cut off the regions where $\rho$ is unbounded. Indeed, this second possibility is encoded in the notion of renormalized solution (9).

The well-posedness theory provided by (9) for bounded densities is sufficient for the existence of a regular Lagrangian flow, which in turn leads to existence also for unbounded densities. For the uniqueness, an additional integrability condition is required:

Theorem 1.1 (DiPerna-Lions [26]) Let $p, \tilde{p} \in[1, \infty]$ and let $u \in L^{1}\left(0, T ; W^{1, \tilde{p}}\left(\mathbb{T}^{d}\right)\right.$ ) be a vector field with div $u=0$. For any $\rho^{0} \in L^{p}\left(\mathbb{T}^{d}\right)$ there exists a unique renormalized solution of (1)-(2), satisfying $\rho \in C\left([0, T] ; L^{p}\left(\mathbb{T}^{d}\right)\right)$. Moreover, if

$$
\frac{1}{p}+\frac{1}{\tilde{p}} \leq 1
$$

then this solution is unique among all weak solutions with $\rho \in L^{\infty}\left(0, T ; L^{p}\left(\mathbb{T}^{d}\right)\right)$. 
As we have already observed for the case of bounded densities, also in this more general setting existence of weak and renormalized solutions is not a difficult problem. It is as well not hard to show uniqueness in the class of renormalized solutions, using the fact that renormalized solutions in $L_{t}^{\infty} L_{x}^{p}$ have constant in time $L^{p}$ norm (it suffices to choose $\beta$ as a bounded smooth approximation of $\tau \mapsto|\tau|^{p}$ ).

The crucial point in Theorem 1.1 concerns the uniqueness of the renormalized solution among all the weak solutions in $L_{t}^{\infty} L_{x}^{p}$, provided (11) is satisfied. The reason why such uniqueness holds can be explained by the same heuristic as in the case of bounded densities: a vector field in $W^{1, \tilde{p}}$ is "Lipschitz-like" except on a small bad set, which can not "be seen" by a density in $L^{p}$, if (11) holds, i.e. if $p$, although it is less than $\infty$, is sufficiently large w.r.t. $\tilde{p}$. On the more technical side, the integrability condition (11) is necessary in the proof in [26] to show convergence of the commutator (10) in $L_{\text {loc }}^{1}$.

The following question is therefore left open: does uniqueness of weak solutions hold in the class of densities $\rho \in L_{t}^{\infty} L_{x}^{p}$ for a vector field in $L_{t}^{1} L_{x}^{p^{\prime}} \cap L_{t}^{1} W_{x}^{1, \tilde{p}}$, when (11) fails?

In a recent note Caravenna and Crippa [15], addressed this issue for the case $p=1$ and $\tilde{p}>1$, announcing the result that uniqueness holds under the additional assumption that $u$ is continuous.

In this paper we show that if

$$
\frac{1}{p}+\frac{1}{\tilde{p}}>1+\frac{1}{d-1}
$$

then, in general, uniqueness fails. We remark that the Sobolev regularity of the vector field $u \in L_{t}^{1} W_{x}^{1, \tilde{p}}$ implies the existence of a unique regular Lagrangian flow (see in particular [7]). Nevertheless, quite surprisingly, our result shows that such Lagrangian uniqueness is of no help to get uniqueness on the Eulerian side.

Previously, examples of such Eulerian non-uniqueness have been constructed, for instance, in [18], based on the method of convex integration from [21], yielding merely bounded velocity $u$ and density $\rho$. However, such examples do not satisfy the differentiability condition $u \in W^{1, \tilde{p}}$ for any $\tilde{p} \geq 1$ and therefore do not possess an associated Lagrangian flow.

Here is the statement of our first and main result.

Theorem 1.2 Let $\varepsilon>0, \bar{\rho} \in C^{\infty}\left([0, T] \times \mathbb{T}^{d}\right)$, with

$$
\int_{\mathbb{T}^{d}} \bar{\rho}(0, x) d x=\int_{\mathbb{T}^{d}} \bar{\rho}(t, x) d x \text { for every } t \in[0, T]
$$

Let $p \in(1, \infty), \tilde{p} \in[1, \infty)$ such that (12) holds. Then there exist $\rho:[0, T] \times \mathbb{T}^{d} \rightarrow \mathbb{R}$, $u:[0, T] \times \mathbb{T}^{d} \rightarrow \mathbb{R}^{d}$ such that

(a) $\rho \in C\left([0, T] ; L^{p}\left(\mathbb{T}^{d}\right)\right), u \in C\left([0, T] ; W^{1, \tilde{p}}\left(\mathbb{T}^{d}\right) \cap L^{p^{\prime}}\left(\mathbb{T}^{d}\right)\right)$;

(b) $(\rho, u)$ is a weak solution to (1) and (3); 
(c) at initial and final time $\rho$ coincides with $\bar{\rho}$, i.e.

$$
\rho(0, \cdot)=\bar{\rho}(0, \cdot), \quad \rho(T, \cdot)=\bar{\rho}(T, \cdot) ;
$$

(d) $\rho$ is $\varepsilon$-close to $\bar{\rho}$ i.e.

$$
\sup _{t \in[0, T]}\|\rho(t, \cdot)-\bar{\rho}(t, \cdot)\|_{L^{p}\left(\mathbb{T}^{d}\right)} \leq \varepsilon .
$$

Our theorem has the following immediate consequences.

Corollary 1.3 (Non-uniqueness) Assume (12). Let $\bar{\rho} \in C^{\infty}\left(\mathbb{T}^{d}\right)$ with $\int_{\mathbb{T}^{d}} \bar{\rho} d x=0$. Then there exist

$$
\left.\rho \in C\left([0, T] ; L^{p}\left(\mathbb{T}^{d}\right)\right), \quad u \in C\left([0, T] ; W^{1, \tilde{p}}\left(\mathbb{T}^{d}\right) \cap L^{p^{\prime}}\left(\mathbb{T}^{d}\right)\right)\right)
$$

such that $(\rho, u)$ is a weak solution to (1), (3), and $\rho \equiv 0$ at $t=0, \rho \equiv \bar{\rho}$ at $t=T$.

Proof Let $\chi:[0, T] \rightarrow \mathbb{R}$ such that $\chi \equiv 0$ on $[0, T / 4], \chi \equiv 1$ on $[3 T / 4, T]$. Apply Theorem 1.2 with $\bar{\rho}(t, x):=\chi(t) \bar{\rho}(x)$.

Corollary 1.4 (Non-renormalized solution) Assume (12). Then there exist

$$
\left.\rho \in C\left([0, T] ; L^{p}\left(\mathbb{T}^{d}\right)\right), \quad u \in C\left([0, T] ; W^{1, \tilde{p}}\left(\mathbb{T}^{d}\right) \cap L^{p^{\prime}}\left(\mathbb{T}^{d}\right)\right)\right)
$$

such that $(\rho, u)$ is a weak solution to (1), (3), and $\|\rho(t)\|_{L^{p}\left(\mathbb{T}^{d}\right)}$ is not constant in time.

Proof Take a smooth map $\bar{\rho}(t, x)$ such that its spatial mean value is constant in time, but its $L^{p}$ norm is not constant in time. Apply Theorem 1.2 with such $\bar{\rho}$ and

$$
\varepsilon:=\frac{1}{4} \max _{t, s}\left|\|\rho(t)\|_{L^{p}\left(\mathbb{T}^{d}\right)}-\|\rho(s)\|_{L^{p}\left(\mathbb{T}^{d}\right)}\right| .
$$

Remark 1.5 We list some remarks about the statement of the theorem.

1. Condition (12) implies that $d \geq 3$. In fact it is not clear if a similar statement could hold for $d=2$ - see for instance [2] for the case of autonomous vector fields.

2. Our theorem shows the optimality of the condition of DiPerna-Lions in (11), at least for sufficiently high dimension $d \geq 3$.

3. The requirement that $\bar{\rho}$ has constant (in time) spatial mean value is necessary because weak solutions to (1), (3) preserve the spatial mean.

4. The condition (12) implies that the $L^{p^{\prime}}$-integrability of the velocity $u$ does not follow from the Sobolev embedding theorem.

5. We expect that the statement of Theorem 1.2 remains valid if (12) is replaced by

$$
\frac{1}{p}+\frac{1}{\tilde{p}}>1+\frac{1}{d}
$$


It would be interesting to see if this condition is sharp in the sense that uniqueness holds provided

$$
\frac{1}{p}+\frac{1}{\tilde{p}} \leq 1+\frac{1}{d}
$$

In this regard we note that (13) implies $\tilde{p}<d$. Conversely, if $u \in W^{1, \tilde{p}}$ with $\tilde{p}>d$, the Sobolev embedding implies that $u$ is continuous so that the uniqueness statement in [15] applies.

6. The given function $\bar{\rho}$ could be less regular than $C^{\infty}$, but we are not interested in following this direction here.

7. It can be shown that the dependence of $\rho, u$ on time is actually $C_{t}^{\infty}$, not just continuous, since we treat time just as a parameter.

Inspired by the heuristic described above, the proof of our theorem is based on the construction of densities $\rho$ and vector fields $u$ so that $\rho$ is, in some sense, concentrated on the "bad" set of $u$, provided (12) holds. To construct such densities and fields, we treat the linear transport equation (1) as a non-linear PDE, whose unknowns are both $\rho$ and $u$ : this allows us to control the interplay between density and field. More precisely, we must deal with two opposite needs: on one side, to produce "anomalous" solutions, we need to highly concentrate $\rho$ and $u$; on the other side, too highly concentrated functions fail to be Sobolev or even $L^{p}$-integrable. The balance between these two needs is expressed by (12).

It is therefore possible to guess that, under a more restrictive assumption than (12), one could produce anomalous solutions enjoying much more regularity than just $\rho \in L^{p}$ and $u \in W^{1, \tilde{p}}$. Indeed, we can produce anomalous solutions as regular as we like, as shown in the next theorem, where (12) is replaced by (14).

Theorem 1.6 Let $\varepsilon>0, \bar{\rho} \in C^{\infty}\left([0, T] \times \mathbb{T}^{d}\right)$, with

$$
\int_{\mathbb{T}^{d}} \bar{\rho}(0, x) d x=\int_{\mathbb{T}^{d}} \bar{\rho}(t, x) d x \text { for every } t \in[0, T] .
$$

Let $p, \tilde{p} \in[1, \infty)$ and $m, \tilde{m} \in \mathbb{N}$ such that

$$
\frac{1}{p}+\frac{1}{\tilde{p}}>1+\frac{m+\tilde{m}}{d-1} .
$$

Then there exist $\rho:[0, T] \times \mathbb{T}^{d} \rightarrow \mathbb{R}, u:[0, T] \times \mathbb{T}^{d} \rightarrow \mathbb{R}^{d}$ such that

(a) $\rho \in C\left([0, T], W^{m, p}\left(\mathbb{T}^{d}\right)\right), u \in C\left([0, T] ; W^{\tilde{m}, \tilde{p}}\left(\mathbb{T}^{d}\right)\right), \rho u \in C\left([0,1] ; L^{1}\left(\mathbb{T}^{d}\right)\right)$;

(b) $(\rho, u)$ is a weak solution to (1), (3);

(c) at initial and final time $\rho$ coincides with $\bar{\rho}$, i.e.

$$
\rho(0, \cdot)=\bar{\rho}(0, \cdot), \quad \rho(T, \cdot)=\bar{\rho}(T, \cdot) ;
$$

(d) $\rho$ is $\varepsilon$-close to $\bar{\rho}$ i.e.

$$
\sup _{t \in[0, T]}\|\rho(t, \cdot)-\bar{\rho}(t, \cdot)\|_{W^{m, p}\left(\mathbb{T}^{d}\right)} \leq \varepsilon .
$$


Remark 1.7 The analogues of Corollaries 1.3 and 1.4 continue to hold in Theorems 1.6. Observe also that (14) reduces to (12) if we choose $m=0$ and $\tilde{m}=1$.

Remark 1.8 Contrary to Theorem 1.2, here we do not show that $u \in C\left([0, T], L^{p^{\prime}}\left(\mathbb{T}^{d}\right)\right)$. Here we prove that $\rho u \in C\left([0, T], L^{1}\left(\mathbb{T}^{d}\right)\right)$ by showing that $\rho \in C\left([0, T] ; L^{s}\left(\mathbb{T}^{d}\right)\right)$ and $u \in C\left([0, T] ; L^{s^{\prime}}\left(\mathbb{T}^{d}\right)\right)$ for some suitably chosen $s, s^{\prime} \in(1, \infty)$. This is also the reason why in Theorem 1.6 we allow the case $p=1$. Indeed, Theorem 1.2, for any given $p$, produces a vector field $u \in C_{t} L_{x}^{p^{\prime}}$; on the contrary, Theorem 1.6 just produces a field $u \in C_{t} L_{x}^{s^{\prime}}$, for some $s^{\prime}<p^{\prime}$.

\subsection{Extension to the Transport-Diffusion Equation}

The mechanism of concentrating the density in the same set where the field is concentrated, used to construct anomalous solutions to the transport equation, can be used as well to prove non-uniqueness for the transport-diffusion equation (7).

The diffusion term $\Delta \rho$ "dissipates the energy" and therefore, heuristically, it helps for uniqueness. Non-uniqueness can thus be caused only by the transport term $\operatorname{div}(\rho u)=u \cdot \nabla \rho$. Therefore, as a general principle, whenever a uniqueness result is available for the transport equation, the same result applies to the transport-diffusion equation (see, for instance, $[19,32,34]$ ). Moreover, the diffusion term $\Delta \rho$ is so strong that minimal assumptions on $u$ are enough to have uniqueness: this is the case, for instance, if $u$ is just bounded, or even $u \in L_{t}^{r} L_{x}^{q}$, with $2 / r+d / q \leq 1$ (see [31] and also [9], where this relation between $r, q, d$ is proven to be sharp). Essentially, in this regime the transport term can be treated as a lower order perturbation of the heat equation.

On the other hand, the technique we use to prove non-uniqueness for the transport equation allows us to construct densities and fields, whose concentrations are so high that the transport term "wins" over the diffusion one and produces anomalous solutions to (7) as well. Roughly speaking, we have to compare $\operatorname{div}(\rho u)$ with $\Delta \rho=\operatorname{div}(\nabla \rho)$, or, equivalently, $\rho u$ with $\nabla \rho$, for instance in the $L^{1}$ norm. The way we construct concentration of $\rho$ and $u$ can be arranged, under a more restrictive assumption than (12), so that

$$
\|\rho u\|_{L^{1}} \approx 1, \quad\|\nabla \rho\|_{L^{1}} \ll 1
$$

[see the last inequality in (37) and (51)] and thus the transport term is "much larger" than the diffusion one. The precise statement is as follows.

Theorem 1.9 Let $\varepsilon>0, \bar{\rho} \in C^{\infty}\left([0, T] \times \mathbb{T}^{d}\right)$, with

$$
\int_{\mathbb{T}^{d}} \bar{\rho}(0, x) d x=\int_{\mathbb{T}^{d}} \bar{\rho}(t, x) d x \text { for every } t \in[0, T] .
$$

Let $p \in(1, \infty), \tilde{p} \in[1, \infty)$ such that

$$
\frac{1}{p}+\frac{1}{\tilde{p}}>1+\frac{1}{d-1}, \quad p^{\prime}<d-1
$$


Then there exist $\rho:[0, T] \times \mathbb{T}^{d} \rightarrow \mathbb{R}, u:[0, T] \times \mathbb{T}^{d} \rightarrow \mathbb{R}^{d}$ such that

(a) $\rho \in C\left([0, T] ; L^{p}\left(\mathbb{T}^{d}\right)\right), u \in C\left([0, T] ; W^{1, \tilde{p}}\left(\mathbb{T}^{d}\right) \cap L^{p^{\prime}}\left(\mathbb{T}^{d}\right)\right)$;

(b) $(\rho, u)$ is a weak solution to (7) and (3);

(c) at initial and final time $\rho$ coincides with $\bar{\rho}$, i.e.

$$
\rho(0, \cdot)=\bar{\rho}(0, \cdot), \quad \rho(T, \cdot)=\bar{\rho}(T, \cdot) ;
$$

(d) $\rho$ is $\varepsilon$-close to $\bar{\rho}$ i.e.

$$
\sup _{t \in[0, T]}\|\rho(t, \cdot)-\bar{\rho}(t, \cdot)\|_{L^{p}\left(\mathbb{T}^{d}\right)} \leq \varepsilon .
$$

As for the transport equation, also for (7) we can generalize Theorem 1.9, to get densities and fields with arbitrary large regularity. Moreover, we can cover also the case of diffusion operators of arbitrary large order:

$$
\partial_{t} \rho+\operatorname{div}(\rho u)=L \rho
$$

where $L$ is a constant coefficient differential operator of order $k \in \mathbb{N}, k \geq 2$, not necessarily elliptic.

Theorem 1.10 Let $\varepsilon>0, \bar{\rho} \in C^{\infty}\left([0, T] \times \mathbb{T}^{d}\right)$, with

$$
\int_{\mathbb{T}^{d}} \bar{\rho}(0, x) d x=\int_{\mathbb{T}^{d}} \bar{\rho}(t, x) d x \text { for every } t \in[0, T] .
$$

Let $p, \tilde{p} \in[1, \infty)$ and $m, \tilde{m} \in \mathbb{N}$ such that

$$
\frac{1}{p}+\frac{1}{\tilde{p}}>1+\frac{m+\tilde{m}}{d-1}, \quad \tilde{p}<\frac{d-1}{\tilde{m}+k-1} .
$$

Then there exist $\rho:[0, T] \times \mathbb{T}^{d} \rightarrow \mathbb{R}, u:[0, T] \times \mathbb{T}^{d} \rightarrow \mathbb{R}^{d}$ such that

(a) $\rho \in C\left([0, T] ; W^{m, p}\left(\mathbb{T}^{d}\right)\right), u \in C\left([0, T] ; W^{\tilde{m}, \tilde{p}}\left(\mathbb{T}^{d}\right)\right), \rho u \in C\left([0,1] ; L^{1}\left(\mathbb{T}^{d}\right)\right)$;

(b) $(\rho, u)$ is a weak solution to (16) and (3);

(c) at initial and final time $\rho$ coincides with $\bar{\rho}$, i.e.

$$
\rho(0, \cdot)=\bar{\rho}(0, \cdot), \quad \rho(T, \cdot)=\bar{\rho}(T, \cdot) ;
$$

(d) $\rho$ is $\varepsilon$-close to $\bar{\rho}$ i.e.

$$
\sup _{t \in[0, T]}\|\rho(t, \cdot)-\bar{\rho}(t, \cdot)\|_{W^{m, p}\left(\mathbb{T}^{d}\right)} \leq \varepsilon
$$

Remark 1.11 The analogues of Corollaries 1.3 and 1.4 continue to hold in Theorems 1.9 and 1.10. Remark 1.8 applies also to the statement of Theorem 1.10.

Observe also that, if we choose $m=0, \tilde{m}=1, k=2$, the first condition in (15) reduces to the first condition in (17), nevertheless (15) is not equivalent to (17). Indeed, 
(15) implies (17), but the viceversa is not true, in general. This can be explained by the fact that Theorem 1.9, for any given $p$, produces a vector field $u \in C_{t} L_{x}^{p^{\prime}}$, while Theorem 1.10 just produces a field $u \in C_{t} L_{x}^{s^{\prime}}$ for some $s^{\prime}<p^{\prime}$.

\subsection{Strategy of the Proof}

Our strategy is based on the technique of convex integration that has been developed in the past years for the incompressible Euler equations in connection with Onsager's conjecture, see [10-13,22,23,29] and in particular inspired by the recent extension of the techniques to weak solutions of the Navier-Stokes equations in [14]. Whilst the techniques that led to progress and eventual resolution of Onsager's conjecture in [29] are suitable for producing examples with Hölder continuous velocity (with small exponent) [30], being able to ensure that the velocity is in a Sobolev space $W^{1, \tilde{p}}$, i.e. with one full derivative, requires new ideas.

A similar issue appears when one wants to control the dissipative term $-\Delta u$ in the Navier-Stokes equations. Inspired by the theory of intermittency in hydrodynamic turbulence, Buckmaster and Vicol [14] introduced "intermittent Beltrami flows", which are spatially inhomogeneous versions of the classical Beltrami flows used in [10-12,22,23]. In contrast to the homogeneous case, these have different scaling for different $L^{q}$ norms at the expense of a diffuse Fourier support. In particular, one can ensure small $L^{q}$ norm for small $q>1$, which in turn leads to control of the dissipative term.

In this paper we introduce concentrations to the convex integration scheme in a different way, closer in spirit to the $\beta$-model, introduced by Frisch et al. $[27,28]$ as a simple model for intermittency in turbulent flows. In addition to a large parameter $\lambda$ that controls the frequency of oscillations, we introduce a second large parameter $\mu$ aimed at controlling concentrations. Rather than working in Fourier space, we work entirely in $x$-space and use "Mikado flows", introduced in [20] and used in $[13,29]$ as the basic building blocks. These building blocks consist of pairwise disjoint (periodic) pipes in which the divergence-free velocity and, in our case, the density are supported. In particular, our construction only works for dimensions $d \geq 3$. The oscillation parameter $\lambda$ controls the frequency of the periodic arrangement - the pipes are arranged periodically with period $1 / \lambda$. The concentration parameter $\mu$ controls the relative (to $1 / \lambda$ ) radius of the pipes and the size of the velocity and density. Thus, for large $\mu$ our building blocks consist of a $1 / \lambda$-periodic arrangement of very thin pipes of total volume fraction $1 / \mu^{d-1}$ where the velocity and density are concentrated-see Proposition 4.1 and Remark 4.2 below.

We prove in details only Theorem 1.2, in Sects. 2-6. The proofs of Theorems $1.6,1.9,1.10$ can be obtained from the one of Theorem 1.2 with minor changes. A sketch is provided in Sect. 7.

\section{Technical Tools}

We start by fixing some notation: 
- $\mathbb{T}^{d}=\mathbb{R}^{d} / \mathbb{Z}^{d}$ is the $d$-dimensional flat torus.

- For $p \in[1, \infty]$ we will always denote by $p^{\prime}$ its dual exponent.

- If $f(t, x)$ is a smooth function of $t \in[0, T]$ and $x \in \mathbb{T}^{d}$, we denote by

- $\|f\|_{C^{k}}$ the sup norm of $f$ together with the sup norm of all its derivatives in time and space up to order $k$;

- $\|f(t, \cdot)\|_{C^{k}\left(\mathbb{T}^{d}\right)}$ the sup norm of $f$ together with the sup norm of all its spatial derivatives up to order $k$ at fixed time $t$;

- $\|f(t, \cdot)\|_{L^{p}\left(\mathbb{T}^{d}\right)}$ the $L^{p}$ norm of $f$ in the spatial derivatives, at fixed time $t$. Since we will take always $L^{p}$ norms in the spatial variable (and never in the time variable), we will also use the shorter notation $\|f(t, \cdot)\|_{L^{p}}=\|f(t)\|_{L^{p}}$ to denote the $L^{p}$ norm of $f$ in the spatial variable.

- $C_{0}^{\infty}\left(\mathbb{T}^{d}\right)$ is the set of smooth functions on the torus with zero mean value.

- $\mathbb{N}=\{0,1,2, \ldots\}$.

- We will use the notation $C\left(A_{1}, \ldots, A_{n}\right)$ to denote a constant which depends only on the numbers $A_{1}, \ldots, A_{n}$.

We now introduce three technical tools, namely an improved Hölder inequality, an antidivergence operator and a lemma about the mean value of fast oscillating functions. These tools will be frequently used in the following. For a function $g \in C^{\infty}\left(\mathbb{T}^{d}\right)$ and $\lambda \in \mathbb{N}$, we denote by $g_{\lambda}: \mathbb{T}^{d} \rightarrow \mathbb{R}$ the $1 / \lambda$ periodic function defined by

$$
g_{\lambda}(x):=g(\lambda x)
$$

Notice that for every $k \in \mathbb{N}$ and $p \in[1, \infty]$

$$
\left\|D^{k} g_{\lambda}\right\|_{L^{p}\left(\mathbb{T}^{d}\right)}=\lambda^{k}\left\|D^{k} g\right\|_{L^{p}\left(\mathbb{T}^{d}\right)} .
$$

\subsection{Improved Hölder Inequality}

We start with the statement of the improved Hölder inequality, inspired by Lemma 3.7 in [14].

Lemma 2.1 Let $\lambda \in \mathbb{N}$ and $f, g: \mathbb{T}^{d} \rightarrow \mathbb{R}$ be smooth functions. Then for every $p \in[1, \infty]$,

$$
\left|\left\|f g_{\lambda}\right\|_{L^{p}}-\|f\|_{L^{p}}\|g\|_{L^{p}}\right| \leq \frac{C_{p}}{\lambda^{1 / p}}\|f\|_{C^{1}}\|g\|_{L^{p}},
$$

where all the norms are taken on $\mathbb{T}^{d}$. In particular

$$
\left\|f g_{\lambda}\right\|_{L^{p}} \leq\|f\|_{L^{p}}\|g\|_{L^{p}}+\frac{C_{p}}{\lambda^{1 / p}}\|f\|_{C^{1}}\|g\|_{L^{p}}
$$


Proof Let us divide $\mathbb{T}^{d}$ into $\lambda^{d}$ small cubes $\left\{Q_{j}\right\}_{j}$ of edge $1 / \lambda$. On each $Q_{j}$ we have

$$
\begin{aligned}
\int_{Q_{j}} & |f(x)|^{p}\left|g_{\lambda}(x)\right|^{p} \\
\quad & =\int_{Q_{j}}\left(|f(x)|^{p}-f_{Q_{j}}|f(y)|^{p} d y\right)\left|g_{\lambda}(x)\right|^{p} d x+f_{Q_{j}}|f(y)|^{p} d y \int_{Q_{j}}\left|g_{\lambda}(x)\right|^{p} d x \\
& \left(\text { since } g_{\lambda}(x)=g(\lambda x)\right) \\
& =\int_{Q_{j}}\left(|f(x)|^{p}-f_{Q_{j}}|f(y)|^{p} d y\right)\left|g_{\lambda}(x)\right|^{p} d x+\frac{1}{\lambda^{d}} f_{Q_{j}}|f(y)|^{p} d y \int_{\mathbb{T}^{d}}|g(x)|^{p} d x \\
& \left(\operatorname{since}\left|Q_{j}\right|=1 / \lambda^{d}\right) \\
& =\int_{Q_{j}}\left(|f(x)|^{p}-f_{Q_{j}}|f(y)|^{p} d y\right)\left|g_{\lambda}(x)\right|^{p} d x+\int_{Q_{j}}|f(y)|^{p} d y \int_{\mathbb{T}^{d}}|g(x)|^{p} d x
\end{aligned}
$$

Summing over $j$ we get

$$
\begin{aligned}
\left\|f g_{\lambda}\right\|_{L^{p}\left(\mathbb{T}^{d}\right)}^{p}= & \|f\|_{L^{p}\left(\mathbb{T}^{d}\right)}^{p}\|g\|_{L^{p}\left(\mathbb{T}^{d}\right)}^{p} \\
& +\sum_{j} \int_{Q_{j}}\left(|f(x)|^{p}-f_{Q_{j}}|f(y)|^{p} d y\right)\left|g_{\lambda}(x)\right|^{p} d x .
\end{aligned}
$$

Let us now estimate the second term in the r.h.s. For $x, y \in Q_{j}$ it holds

$$
\left.|| f(x)\right|^{p}-|f(y)|^{p} \mid \leq \frac{C_{p}}{\lambda}\|f\|_{C^{0}\left(\mathbb{T}^{d}\right)}^{p-1}\|\nabla f\|_{C^{0}\left(\mathbb{T}^{d}\right)} \leq \frac{C_{p}}{\lambda}\|f\|_{C^{1}\left(\mathbb{T}^{d}\right)}^{p}
$$

Therefore

$$
\begin{aligned}
& \sum_{j} \int_{Q_{j}}\left(|f(x)|^{p}-f_{Q_{j}}|f(y)|^{p} d y\right)\left|g_{\lambda}(x)\right|^{p} d x \\
& \leq \frac{C_{p}}{\lambda}\|f\|_{C^{1}\left(\mathbb{T}^{d}\right)}^{p} \sum_{j} \int_{Q_{j}}\left|g_{\lambda}(x)\right|^{p} d x \\
& =\frac{C_{p}}{\lambda}\|f\|_{C^{1}\left(\mathbb{T}^{d}\right)}^{p}\left\|g_{\lambda}\right\|_{L^{p}\left(\mathbb{T}^{d}\right)}^{p} \\
& =\frac{C_{p}}{\lambda}\|f\|_{C^{1}\left(\mathbb{T}^{d}\right)}^{p}\|g\|_{L^{p}\left(\mathbb{T}^{d}\right)}^{p},
\end{aligned}
$$

from which we get

$$
\left|\left\|f g_{\lambda}\right\|_{L^{p}}^{p}-\|f\|_{L^{p}}^{p}\|g\|_{L^{p}}^{p}\right| \leq \frac{C_{p}}{\lambda}\|f\|_{C^{1}}^{p}\|g\|_{L^{p}}^{p}
$$

Inequality (19) is now obtained by taking the $1 / p$ power in the last formula and using that for $A, B>0,|A-B|^{p} \leq\left.|| A\right|^{p}-|B|^{p} \mid$. Finally, the improved Hölder inequality (20) is an immediate consequence of (19). 


\subsection{Antidivergence Operators}

For $f \in C_{0}^{\infty}\left(\mathbb{T}^{d}\right)$ there exists a unique $u \in C_{0}^{\infty}\left(\mathbb{T}^{d}\right)$ such that $\Delta u=f$. The operator $\Delta^{-1}: C_{0}^{\infty}\left(\mathbb{T}^{d}\right) \rightarrow C_{0}^{\infty}\left(\mathbb{T}^{d}\right)$ is thus well defined. We define the standard antidivergence operator as $\nabla \Delta^{-1}: C_{0}^{\infty}\left(\mathbb{T}^{d}\right) \rightarrow C^{\infty}\left(\mathbb{T}^{d} ; \mathbb{R}^{d}\right)$. It clearly satisfies $\operatorname{div}\left(\nabla \Delta^{-1} f\right)=f$.

Lemma 2.2 For every $k \in \mathbb{N}$ and $p \in[1, \infty]$, the standard antidivergence operator satisfies the bounds

$$
\left\|D^{k}\left(\nabla \Delta^{-1} g\right)\right\|_{L^{p}} \leq C_{k, p}\left\|D^{k} g\right\|_{L^{p}} .
$$

Moreover for every $\lambda \in \mathbb{N}$ it holds

$$
\left\|D^{k}\left(\nabla \Delta^{-1} g_{\lambda}\right)\right\|_{L^{p}} \leq C_{k, p} \lambda^{k-1}\left\|D^{k} g\right\|_{L^{p}}
$$

Proof For $p \in(1, \infty)$ from the Calderon-Zygmund inequality we get

$$
\left\|D^{k}\left(\nabla \Delta^{-1} g\right)\right\|_{W^{1, p}\left(\mathbb{T}^{d}\right)} \leq C_{k, p}\left\|D^{k} g\right\|_{L^{p}\left(\mathbb{T}^{d}\right)}
$$

from which (21) follows. For $p=\infty$, we use Sobolev embeddings to get

$$
\begin{aligned}
\left\|D^{k}\left(\nabla \Delta^{-1} g\right)\right\|_{L^{\infty}\left(\mathbb{T}^{d}\right)} & \leq C\left\|D^{k}\left(\nabla \Delta^{-1} g\right)\right\|_{W^{1, d+1}\left(\mathbb{T}^{d}\right)} \\
\text { (by (23) with } p=d+1) & \leq C_{k, d+1}\left\|D^{k} g\right\|_{L^{d+1}\left(\mathbb{T}^{d}\right)} \\
& \leq C_{k, \infty}\left\|D^{k} g\right\|_{L^{\infty}\left(\mathbb{T}^{d}\right)} .
\end{aligned}
$$

For $p=1$ we use the dual characterization of $L^{1}$ norm. For every $f \in L^{1}$,

$$
\begin{aligned}
\|f\|_{L^{1}\left(\mathbb{T}^{d}\right)} & =\max \left\{\int_{\mathbb{T}^{d}} f \varphi: \varphi \in L^{\infty}\left(\mathbb{T}^{d}\right),\|\varphi\|_{L^{\infty}\left(\mathbb{T}^{d}\right)}=1\right\} \\
& =\sup \left\{\int_{\mathbb{T}^{d}} f \varphi: \varphi \in C^{\infty}\left(\mathbb{T}^{d}\right),\|\varphi\|_{L^{\infty}\left(\mathbb{T}^{d}\right)}=1\right\} .
\end{aligned}
$$

Moreover, if $f_{\mathbb{T}^{d}} f=0$, it also holds

$$
\|f\|_{L^{1}\left(\mathbb{T}^{d}\right)}=\sup \left\{\int_{\mathbb{T}^{d}} f \varphi: \varphi \in C_{0}^{\infty}\left(\mathbb{T}^{d}\right),\|\varphi\|_{L^{\infty}\left(\mathbb{T}^{d}\right)}=1\right\}
$$


Therefore (in the following formula $\partial^{k}$ denotes any partial derivative of order $k$ ):

$$
\begin{aligned}
\left\|\partial^{k}\left(\nabla \Delta^{-1} g\right)\right\|_{L^{1}\left(\mathbb{T}^{d}\right)} & =\sup _{\substack{\varphi \in C_{0}^{\infty}\left(\mathbb{T}^{d}\right) \\
\|\varphi\|_{L^{\infty}=1}}} \int_{\mathbb{T}^{d}} \partial^{k}\left(\nabla \Delta^{-1} g\right) \varphi d x \\
& =\sup _{\substack{\varphi \in C_{0}^{\infty}\left(\mathbb{T}^{d}\right) \\
\|\varphi\|_{L^{\infty}=1}}} \int_{\mathbb{T}^{d}} \partial^{k} g \nabla \Delta^{-1} \varphi d x \\
\text { (by Hölder) } \leq & \sup _{\substack{\varphi \in C_{0}^{\infty}\left(\mathbb{T}^{d}\right) \\
\|\varphi\|_{L^{\infty}=1}}}\left\|\partial^{k} g\right\|_{L^{1}\left(\mathbb{T}^{d}\right)}\left\|\nabla \Delta^{-1} \varphi\right\|_{L^{\infty}\left(\mathbb{T}^{d}\right)} \\
\text { (using (21) with } p=\infty) \leq & C_{0, \infty}\left\|\partial^{k} g\right\|_{L^{1}\left(\mathbb{T}^{d}\right)} \underset{\substack{\varphi \in C_{0}^{\infty}\left(\mathbb{T}^{d}\right) \\
\|\varphi\|_{L^{\infty}=1}}}{\leq} C_{0, \infty}\left\|\partial_{L^{\infty}\left(\mathbb{T}^{d}\right)} g\right\|_{L^{1}\left(\mathbb{T}^{d}\right)},
\end{aligned}
$$

from with (21) with $p=1$ follows. To prove (22), observe that

$$
\nabla \Delta^{-1} g_{\lambda}(x)=\frac{1}{\lambda}\left(\nabla \Delta^{-1} g\right)(\lambda x)
$$

Therefore

$$
\begin{aligned}
\left\|D^{k}\left(\nabla \Delta^{-1} g_{\lambda}\right)\right\|_{L^{p}\left(\mathbb{T}^{d}\right)} & \leq \lambda^{k-1}\left\|\left(D^{k}\left(\nabla \Delta^{-1} g\right)\right)(\lambda \cdot)\right\|_{L^{p}\left(\mathbb{T}^{d}\right)} \\
& \leq \lambda^{k-1}\left\|D^{k}\left(\nabla \Delta^{-1} g\right)\right\|_{L^{p}\left(\mathbb{T}^{d}\right)} \\
(\text { by }(21)) & \leq C_{k, p} \lambda^{k-1}\left\|D^{k} g\right\|_{L^{p}\left(\mathbb{T}^{d}\right)},
\end{aligned}
$$

thus proving (22).

With the help of the standard antidivergence operator, we now define an improved antidivergence operator, which lets us gain a factor $\lambda^{-1}$ when applied to functions of the form $f(x) g(\lambda x)$.

Lemma 2.3 Let $\lambda \in \mathbb{N}$ and $f, g: \mathbb{T}^{d} \rightarrow \mathbb{R}$ be smooth functions with

$$
f_{\mathbb{T}^{d}} f g_{\lambda}=f_{\mathbb{T}^{d}} g=0 .
$$

Then there exists a smooth vector field $u: \mathbb{T}^{d} \rightarrow \mathbb{R}^{d}$ such that div $u=f g_{\lambda}$ and for every $k \in \mathbb{N}$ and $p \in[1, \infty]$,

$$
\left\|D^{k} u\right\|_{L^{p}} \leq C_{k, p} \lambda^{k-1}\|f\|_{C^{k+1}}\|g\|_{W^{k, p}} .
$$

We will write

$$
u=\mathcal{R}\left(f g_{\lambda}\right) .
$$


Remark 2.4 The same result holds if $f, g$ are vector fields and we want to solve the equation div $u=f \cdot g_{\lambda}$, where $\cdot$ denotes the scalar product.

Proof Set

$$
u:=f \nabla \Delta^{-1} g_{\lambda}-\nabla \Delta^{-1}\left(\nabla f \cdot \nabla \Delta^{-1} g_{\lambda}\right) .
$$

It is immediate from the definition that $\operatorname{div} u=f g_{\lambda}$. We show that (24) holds for $k=0,1$. The general case $k \in \mathbb{N}$ can be easily proven by induction. It holds (the constant $C_{0, p}$ can change its value from line to line)

$$
\begin{aligned}
\|u\|_{L^{p}} & \leq\|f\|_{C^{0}}\left\|\nabla \Delta^{-1} g_{\lambda}\right\|_{L^{p}}+\left\|\nabla \Delta^{-1}\left(\nabla f \cdot \nabla \Delta^{-1} g_{\lambda}\right)\right\|_{L^{p}} \\
(\operatorname{by}(21)) & \leq\|f\|_{C^{0}}\left\|\nabla \Delta^{-1} g_{\lambda}\right\|_{L^{p}}+C_{0, p}\|\nabla f\|_{C^{0}}\left\|\nabla \Delta^{-1} g_{\lambda}\right\|_{L^{p}} \\
(\operatorname{by}(22)) & \leq \frac{C_{0, p}}{\lambda}\|f\|_{C^{1}}\|g\|_{L^{p}},
\end{aligned}
$$

so that (24) holds for $k=0$. For $k=1$ we compute

$\partial_{j} u=\partial_{j} f \nabla \Delta^{-1} g_{\lambda}+f \partial_{j} \nabla \Delta^{-1} g_{\lambda}-\nabla \Delta^{-1}\left(\nabla \partial_{j} f \cdot \nabla \Delta^{-1} g_{\lambda}+\nabla f \cdot \partial_{j} \nabla \Delta^{-1} g_{\lambda}\right)$.

Therefore, using again (21) and (22), (the constant $C_{1, p}$ can change its value from line to line)

$$
\begin{aligned}
\left\|\partial_{j} u\right\|_{L^{p}} \leq & C_{1, p}\left[\left\|\partial_{j} f\right\|_{C^{0}}\left\|\nabla \Delta^{-1} g_{\lambda}\right\|_{L^{p}}+\|f\|_{C^{0}}\left\|\partial_{j} \nabla \Delta^{-1} g_{\lambda}\right\|_{L^{p}}\right. \\
& \left.+\left\|\nabla \partial_{j} f\right\|_{C^{0}}\left\|\nabla \Delta^{-1} g_{\lambda}\right\|_{L^{p}}+\|\nabla f\|_{C^{0}}\left\|\partial_{j} \nabla \Delta^{-1} g_{\lambda}\right\|_{L^{p}}\right] \\
\leq & C_{1, p}\left[\frac{1}{\lambda}\|f\|_{C^{1}}\|g\|_{L^{p}}+\|f\|_{C^{0}}\left\|\partial_{j} g\right\|_{L^{p}}\right. \\
& \left.+\frac{1}{\lambda}\|f\|_{C^{2}}\|g\|_{L^{p}}+\|f\|_{C^{1}}\left\|\partial_{j} g\right\|_{L^{p}}\right] \\
\leq & C_{1, p}\left[\|f\|_{C^{1}}\left\|\partial_{j} g\right\|_{L^{p}}+\frac{1}{\lambda}\|f\|_{C^{2}}\|g\|_{L^{p}}\right] \\
\leq & C_{1, p}\|f\|_{C^{2}}\|g\|_{W^{1, p}} .
\end{aligned}
$$

Remark 2.5 Assume $f$ and $g$ are smooth function of $(t, x), t \in[0, T], x \in \mathbb{T}^{d}$. If at each time $t$ they satisfy (in the space variable) the assumptions of Lemma 2.3, then we can apply $\mathcal{R}$ at each time and define

$$
u(t, \cdot):=\mathcal{R}\left(f(t, \cdot) g_{\lambda}(t, \cdot)\right)
$$

where $g_{\lambda}(t, x)=g(t, \lambda x)$. It follows from the definition of $\mathcal{R}$ that $u$ is a smooth function of $(t, x)$. 


\subsection{Mean Value and Fast Oscillations}

Lemma 2.6 Let $\lambda \in \mathbb{N}$ and $f, g: \mathbb{T}^{d} \rightarrow \mathbb{R}$ be smooth functions with

$$
f_{\mathbb{T}^{d}} g(x) d x=0
$$

Then

$$
\left|f_{\mathbb{T}^{d}} f(x) g(\lambda x) d x\right| \leq \frac{\sqrt{d}\|f\|_{C^{1}}\|g\|_{L^{1}}}{\lambda} .
$$

Proof We divide $\mathbb{T}^{d}$ into small cubes $\left\{Q_{j}\right\}$ of edge $1 / \lambda$. For each $Q_{j}$, choose a point $x_{j} \in Q_{j}$. We have

$$
\begin{aligned}
\left|\int_{\mathbb{T}^{d}} f(x) g(\lambda x) d x\right| & =\left|\sum_{j} \int_{Q_{j}} f(x) g(\lambda x) d x\right| \\
& =\left|\sum_{j} \int_{Q_{j}}\left[f(x)-f\left(x_{j}\right)\right] g(\lambda x) d x\right| \\
& \leq \sum_{j} \int_{Q_{j}}\left|f(x)-f\left(x_{j}\right)\right||g(\lambda x)| d x \\
& \leq \frac{\sqrt{d}\|f\|_{C^{1}}\|g\|_{L^{1}\left(\mathbb{T}^{d}\right)}}{\lambda} .
\end{aligned}
$$

\section{Statement of the Main Proposition and Proof of Theorem 1.2}

We assume without loss of generality that $T=1$ and $\mathbb{T}^{d}$ is the periodic extension of the unit cube $[0,1]^{d}$. The following proposition contains the key facts used to prove Theorem 1.2. Let us first introduce the continuity-defect equation:

$$
\left\{\begin{array}{l}
\partial_{t} \rho+\operatorname{div}(\rho u)=-\operatorname{div} R \\
\operatorname{div} u=0
\end{array}\right.
$$

We will call $R$ the defect field. For $\sigma>0$ set $I_{\sigma}=(\sigma, 1-\sigma)$. Recall that we are assuming $p \in(1, \infty)$.

Proposition 3.1 There exists a constant $M>0$ such that the following holds. Let $\eta, \delta, \sigma>0$ and let $\left(\rho_{0}, u_{0}, R_{0}\right)$ be a smooth solution of the continuity-defect equation (25). Then there exists another smooth solution $\left(\rho_{1}, u_{1}, R_{1}\right)$ of (25) such that

$$
\left\|\rho_{1}(t)-\rho_{0}(t)\right\|_{L^{p}\left(\mathbb{T}^{d}\right)} \leq \begin{cases}M \eta\left\|R_{0}(t)\right\|_{L^{1}\left(\mathbb{T}^{d}\right)}^{1 / p}, & t \in I_{\sigma / 2}, \\ 0, & t \in[0,1] \backslash I_{\sigma / 2},\end{cases}
$$




$$
\begin{aligned}
& \left\|u_{1}(t)-u_{0}(t)\right\|_{L^{p^{\prime}}\left(\mathbb{T}^{d}\right)} \leq \begin{cases}M \eta^{-1}\left\|R_{0}(t)\right\|_{L^{1}\left(\mathbb{T}^{d}\right)}^{1 / p^{\prime}}, & t \in I_{\sigma / 2}, \\
0, & t \in[0,1] \backslash I_{\sigma / 2},\end{cases} \\
& \left\|u_{1}(t)-u_{0}(t)\right\|_{W^{1, \tilde{p}}\left(\mathbb{T}^{d}\right)} \leq \delta, \\
& \left\|R_{1}(t)\right\|_{L^{1}\left(\mathbb{T}^{d}\right)} \leq \begin{cases}\delta, & t \in I_{\sigma}, \\
\left\|R_{0}(t)\right\|_{L^{1}\left(\mathbb{T}^{d}\right)}+\delta, & t \in I_{\sigma / 2} \backslash I_{\sigma}, \\
\left\|R_{0}(t)\right\|_{L^{1}\left(\mathbb{T}^{d}\right)}, & t \in[0,1] \backslash I_{\sigma / 2} .\end{cases}
\end{aligned}
$$

Proof of Theorem 1.2 assuming Proposition 3.1 Let $M$ be the constant in Proposition 3.1. Let $\tilde{\varepsilon}>0$ and $\eta>0$ (their precise value will be fixed later, with $\eta$ depending on $\tilde{\varepsilon})$. Let $\sigma_{q}=\delta_{q}:=2^{-q}$ and $I_{q}:=I_{\sigma_{q}}=\left(\sigma_{q}, 1-\sigma_{q}\right)$.

We construct a sequence $\left(\rho_{q}, u_{q}, R_{q}\right)$ of solutions to (25) as follows. Let $\phi_{0}, \phi, \phi_{1}$ : $[0,1] \rightarrow \mathbb{R}$ three smooth functions such that

$$
\phi_{0}(t)+\phi(t)+\phi_{1}(t)=1 \text { for every } t \in[0,1]
$$

and

$$
\begin{aligned}
\phi_{0}(t) & =1 \text { on }[0, \tilde{\varepsilon}], \\
\phi(t) & =1 \text { on }[2 \tilde{\varepsilon}, 1-2 \tilde{\varepsilon}], \\
\phi_{1}(t) & =1 \text { on }[1-\tilde{\varepsilon}, 1] .
\end{aligned}
$$

Set

$$
\begin{aligned}
& \rho_{0}(t):=\phi_{0}(t) \bar{\rho}(0)+\phi(t) \bar{\rho}(t)+\phi_{1}(t) \bar{\rho}(1), \\
& u_{0}(t):=0, \\
& R_{0}(t):=-\nabla \Delta^{-1}\left(\partial_{t} \rho_{0}(t)+\operatorname{div}\left(\rho_{0}(t) u_{0}(t)\right)\right)=-\nabla \Delta^{-1}\left(\partial_{t} \rho_{0}(t)\right),
\end{aligned}
$$

where the antidivergence is taken with respect to the spatial variable.

Assume now that $\left(\rho_{q}, u_{q}, R_{q}\right)$ is defined. Let $\left(\rho_{q+1}, u_{q+1}, R_{q+1}\right)$ be the solution to the continuity-defect equation, which is obtained by applying Proposition 3.1 to $\left(\rho_{q}, u_{q}, R_{q}\right), \eta$,

$$
\delta=\delta_{q+2}, \quad \sigma=\sigma_{q+1} \quad\left(\text { and thus } \sigma / 2=\sigma_{q+2}\right) .
$$

Lemma 3.2 The following inductive estimates are satisfied:

$$
\left\|\rho_{q}(t)-\rho_{q-1}(t)\right\|_{L^{p}} \leq \begin{cases}M \eta \delta_{q}^{1 / p}, & t \in I_{q-1}, \\ M \eta\left[\left\|R_{0}(t)\right\|_{L^{1}}+\delta_{q}\right]^{1 / p}, & t \in I_{q} \backslash I_{q-1}, \\ M \eta\left\|R_{0}(t)\right\|_{L^{1}}^{1 / p}, & t \in I_{q+1} \backslash I_{q}, \\ 0, & t \in[0,1] \backslash I_{q+1},\end{cases}
$$




$$
\begin{aligned}
&\left\|u_{q}(t)-u_{q-1}(t)\right\|_{L^{p^{\prime}}} \leq \begin{cases}M \eta^{-1} \delta_{q}^{1 / p^{\prime},} & t \in I_{q-1}, \\
M \eta^{-1}\left[\left\|R_{0}(t)\right\|_{L^{1}}+\delta_{q}\right]^{1 / p^{\prime}}, & t \in I_{q} \backslash I_{q-1}, \\
M \eta^{-1}\left\|R_{0}(t)\right\|_{L^{1}}^{1 / p^{\prime}}, & t \in I_{q+1} \backslash I_{q}, \\
0, & t \in[0,1] \backslash I_{q+1},\end{cases} \\
&\left\|u_{q}(t)-u_{q-1}(t)\right\|_{W^{1, \tilde{p}}} \leq \delta_{q+1}, \\
&\left\|R_{q}(t)\right\|_{L^{1}} \leq \begin{cases}\delta_{q+1}, & t \in I_{q}, \\
\left\|R_{0}(t)\right\|_{L^{1}}+\delta_{q+1}, & t \in I_{q+1} \backslash I_{q}, \\
\left\|R_{0}(t)\right\|_{L^{1}}, & t \in[0,1] \backslash I_{q+1} .\end{cases}
\end{aligned}
$$

Proof For $q=0,(28 \mathrm{a})_{q}-(28 \mathrm{c})_{q}$ do not apply, whereas $(28 \mathrm{~d})_{q}$ is trivially satisfied, since $I_{0}=\emptyset$. Assume now that $(28 \mathrm{a})_{q}-(28 \mathrm{~d})_{q}$ hold and let us prove $(28 \mathrm{a})_{q+1}-$ $(28 d)_{q+1}$. From (26a) we get

$$
\left\|\rho_{q+1}(t)-\rho_{q}(t)\right\|_{L^{p}} \leq \begin{cases}M \eta\left\|R_{q}(t)\right\|_{L^{1}}^{1 / p}, & t \in I_{q+2} \\ 0, & t \in[0,1] \text { setminus } I_{q+2}\end{cases}
$$

Therefore, using the inductive assumption $(28 \mathrm{~d})_{q}$, we get:

- if $t \in I_{q}$,

$$
\left\|\rho_{q+1}(t)-\rho_{q}(t)\right\|_{L^{p}} \leq M \eta\left\|R_{q}(t)\right\|_{L^{1}}^{1 / p}, \leq M \eta \delta_{q+1}^{1 / p} ;
$$

- if $t \in I_{q+1} \backslash I_{q}$,

$$
\left\|\rho_{q+1}(t)-\rho_{q}(t)\right\|_{L^{p}} \leq M \eta\left\|R_{q}(t)\right\|_{L^{1}}^{1 / p} \leq M \eta\left[\mid R_{0}(t) \|_{L^{1}}+\delta_{q+1}\right]^{1 / p}
$$

- if $t \in I_{q+2} \backslash I_{q+1}$,

$$
\left\|\rho_{q+1}(t)-\rho_{q}(t)\right\|_{L^{p}} \leq M \eta\left\|R_{q}(t)\right\|_{L^{1}}^{1 / p} \leq M \eta\left\|R_{0}(t)\right\|_{L^{1}}^{1 / p},
$$

and thus $(28 \mathrm{a})_{q+1}$ holds. Estimate $(28 \mathrm{~b})_{q+1}$ can be proven similarly. Estimate $(28 \mathrm{c})_{q+1}$ is an immediate consequence of (26c). Finally, from (26d), we get

$$
\left\|R_{q+1}(t)\right\|_{L^{1}} \leq \begin{cases}\delta_{q+2}, & t \in I_{q+1}, \\ \left\|R_{q}(t)\right\|_{L^{1}}+\delta_{q+2}, & t \in I_{q+2} \backslash I_{q+1}, \\ \left\|R_{q}(t)\right\|_{L^{1}}, & t \in[0,1] \backslash I_{q+2} .\end{cases}
$$

Therefore, using the inductive assumption $(28 \mathrm{~d})_{q}$, we get:

- if $t \in I_{q+2} \backslash I_{q+1}$,

$$
\left\|R_{q+1}(t)\right\|_{L^{1}} \leq\left\|R_{q}(t)\right\|_{L^{1}}+\delta_{q+2} \leq\left\|R_{0}(t)\right\|_{L^{1}}+\delta_{q+2} ;
$$


- if $t \in[0,1] \backslash I_{q+2}$,

$$
\left\|R_{q+1}(t)\right\|_{L^{1}} \leq\left\|R_{q}(t)\right\|_{L^{1}} \leq\left\|R_{0}(t)\right\|_{L^{1}}
$$

from which $(28 \mathrm{~d})_{q+1}$ follows.

It is now an immediate consequence of the previous lemma that there exists

$$
\rho \in C\left((0,1) ; L^{p}\left(\mathbb{T}^{d}\right)\right), \quad u \in C\left((0,1) ; W^{1, \tilde{p}}\left(\mathbb{T}^{d}\right)\right) \cap C\left((0,1) ; L^{p^{\prime}}\left(\mathbb{T}^{d}\right)\right)
$$

such that for every compact subset $K \subseteq(0,1)$

$$
\begin{gathered}
\max _{t \in K}\left\|\rho_{q}(t)-\rho(t)\right\|_{L^{p}} \rightarrow 0 \\
\max _{t \in K}\left\|u_{q}(t)-u(t)\right\|_{L^{p^{\prime}}} \rightarrow 0 \\
\max _{t \in K}\left\|u_{q}(t)-u(t)\right\|_{W^{1, \tilde{p}}} \rightarrow 0 \\
\max _{t \in K}\left\|R_{q}(t)\right\|_{L^{1}} \rightarrow 0,
\end{gathered}
$$

as $q \rightarrow \infty$, from which it follows that $\rho, u$ solves (3)-(4) [or (1)-(3)] in the sense of distributions. This proves part (b) of the statement.

We need now the following estimate. Let $t \in(0,1)$ and let $q^{*}=q^{*}(t) \in \mathbb{N}$ so that $t \in I_{q^{*}} \backslash I_{q^{*-1}}$. By the inductive estimate (28a),

$$
\begin{aligned}
\left\|\rho(t)-\rho_{0}(t)\right\|_{L^{p}} \leq & \sum_{q=1}^{\infty}\left\|\rho_{q}(t)-\rho_{q-1}(t)\right\|_{L^{p}} \\
= & \left\|\rho_{q^{*}-1}(t)-\rho_{q^{*-2}}(t)\right\|_{L^{p}}+\left\|\rho_{q^{*}}(t)-\rho_{q^{*}-1}(t)\right\|_{L^{p}} \\
& +\sum_{q=q^{*}+1}^{\infty}\left\|\rho_{q}(t)-\rho_{q-1}(t)\right\|_{L^{p}} \\
\leq & M \eta\left[\left\|R_{0}(t)\right\|_{L^{1}}^{1 / p}+\left(\left\|R_{0}(t)\right\|_{L^{1}}+\delta_{q^{*}}\right)^{1 / p}+\sum_{q=q^{*}+1}^{\infty} \delta_{q}^{1 / p}\right]
\end{aligned}
$$

Let us now prove that $\|\rho(t)-\bar{\rho}(0)\|_{L^{p}} \rightarrow 0$ as $t \rightarrow 0$. Observe that, for $t<\tilde{\varepsilon}$, $\rho_{0}(t)=\bar{\rho}(0)$ and $R_{0}(t)=0$. Hence, if $t<\tilde{\varepsilon}$,

$$
\begin{aligned}
\|\rho(t)-\bar{\rho}(0)\|_{L^{p}} & =\left\|\rho(t)-\rho_{0}(t)\right\|_{L^{p}} \\
(\operatorname{by}(30)) & \leq M \eta\left[\left\|R_{0}(t)\right\|_{L^{1}}^{1 / p}+\left(\left\|R_{0}(t)\right\|_{L^{1}}+\delta_{q^{*}}\right)^{1 / p}+\sum_{q=q^{*}+1}^{\infty} \delta_{q}^{1 / p}\right] \\
& =M \eta \sum_{q=q^{*}}^{\infty} \delta_{q}^{1 / p},
\end{aligned}
$$


and the conclusion follows observing that $q^{*}=q^{*}(t) \rightarrow \infty$ as $t \rightarrow 0$. In a similar way the limit $\|\rho(t)-\bar{\rho}(1)\|_{L^{p}} \rightarrow 0$ as $t \rightarrow 1$ can be shown. This completes the proof of parts (a) and (c) of the statement.

Let us now prove part (d). We first observe that, for the $\varepsilon$ given in the statement of the theorem, we can choose $\tilde{\varepsilon}$ small enough, so that for every $t \in[0,1]$,

$$
\left\|\rho_{0}(t)-\bar{\rho}(t)\right\|_{L^{p}} \leq \frac{\varepsilon}{2}
$$

Indeed, if $t \in[2 \tilde{\varepsilon}, 1-2 \tilde{\varepsilon}]$, then $\rho_{0}(t)=\bar{\rho}(t)$. If $t \in[0,2 \tilde{\varepsilon}] \cup[1-2 \tilde{\varepsilon}, 1]$, then

$$
\left\|\rho_{0}(t)-\bar{\rho}(t)\right\|_{L^{p}} \leq\left|\phi_{0}(t)\right|\|\bar{\rho}(0)-\bar{\rho}(t)\|_{L^{p}}+\left|\phi_{1}(t)\right|\|\bar{\rho}(1)-\bar{\rho}(t)\|_{L^{p}} \leq \frac{\varepsilon}{2},
$$

where the last inequality follows, by choosing $\tilde{\varepsilon}$ sufficiently small. Therefore, for every $t \in[0,1]$,

$$
\begin{aligned}
\|\rho(t)-\bar{\rho}(t)\|_{L^{p}} & \leq\left\|\rho(t)-\rho_{0}(t)\right\|_{L^{p}}+\left\|\rho_{0}(t)-\bar{\rho}(t)\right\|_{L^{p}} \\
(\operatorname{by}(30)) & \leq M \eta\left[\left\|R_{0}(t)\right\|_{L^{1}}^{1 / p}+\left(\left\|R_{0}(t)\right\|_{L^{1}}+\delta_{q^{*}}\right)^{1 / p}+\sum_{q=q^{*}+1}^{\infty} \delta_{q}^{1 / p}\right]+\frac{\varepsilon}{2} \\
& \leq M \eta \max _{t \in[0,1]}\left[\left\|R_{0}(t)\right\|_{L^{1}}^{1 / p}+\left(\left\|R_{0}(t)\right\|_{L^{1}}+1\right)^{1 / p}+\sum_{q=1}^{\infty} \delta_{q}^{1 / p}\right]+\frac{\varepsilon}{2} \\
& \leq \varepsilon
\end{aligned}
$$

if $\eta$ is chosen small enough (depending on $R_{0}$ and thus on $\tilde{\varepsilon}$ ). This proves part (d) of the statement, thus concluding the proof of the theorem.

\section{The Perturbations}

In this and the next two sections we prove Proposition 3.1. In particular in this section we fix the constant $M$ in the statement of the proposition, we define the functions $\rho_{1}$ and $u_{1}$ and we prove some estimates on them. In Sect. 5 we define $R_{1}$ and we prove some estimates on it. In Sect. 6 we conclude the proof of Proposition 3.1, by proving estimates (26a)-(26d).

\subsection{Mikado Fields and Mikado Densities}

The first step towards the definition of $\rho_{1}, u_{1}$ is the construction of Mikado fields and Mikado densities.

We start by fixing a function $\Phi \in C_{c}^{\infty}\left(\mathbb{R}^{d-1}\right)$ such that

$$
\operatorname{supp} \Phi \subseteq(0,1)^{d-1}, \quad \int_{\mathbb{R}^{d-1}} \Phi=0, \quad \int_{\mathbb{R}^{d-1}} \Phi^{2}=1
$$


Let $\Phi_{\mu}(x):=\Phi(\mu x)$ for $\mu>0$. Let $a \in \mathbb{R}$. For every $k \in \mathbb{N}$, it holds

$$
\left\|D^{k}\left(\mu^{a} \Phi_{\mu}\right)\right\|_{L^{r}\left(\mathbb{R}^{d-1}\right)}=\mu^{a+k-(d-1) / r}\left\|D^{k} \Phi\right\|_{L^{r}\left(\mathbb{R}^{d-1}\right)} .
$$

Proposition 4.1 Let $a, b \in \mathbb{R}$ with

$$
a+b=d-1
$$

For every $\mu>2 d$ and $j=1, \ldots, d$ there exist a Mikado density $\Theta_{\mu}^{j}: \mathbb{T}^{d} \rightarrow \mathbb{R}$ and $a$ Mikado field $W_{\mu}^{j}: \mathbb{T}^{d} \rightarrow \mathbb{R}^{d}$ with the following properties.

(a) It holds

$$
\begin{cases}\operatorname{div} W_{\mu}^{j} & =0, \\ \operatorname{div}\left(\Theta_{\mu}^{j} W_{\mu}^{j}\right) & =0, \\ f_{\mathbb{T}^{d}} \Theta_{\mu}^{j}=f_{\mathbb{T}^{d}} W_{\mu}^{j} & =0, \\ f_{\mathbb{T}^{d}} \Theta_{\mu}^{j} W_{\mu}^{j} & =e_{j},\end{cases}
$$

where $\left\{e_{j}\right\}_{j=1, \ldots, d}$ is the standard basis in $\mathbb{R}^{d}$.

(b) For every $k \in \mathbb{N}$ and $r \in[1, \infty]$

$$
\begin{aligned}
\left\|D^{k} \Theta_{\mu}^{j}\right\|_{L^{r}\left(\mathbb{T}^{d}\right)} \leq\|\Phi\|_{L^{r}\left(\mathbb{R}^{d-1}\right)} \mu^{a+k-(d-1) / r}, \\
\left\|D^{k} W_{\mu}^{j}\right\|_{L^{r}\left(\mathbb{T}^{d}\right)} \leq\|\Phi\|_{L^{r}\left(\mathbb{R}^{d-1}\right)} \mu^{b+k-(d-1) / r},
\end{aligned}
$$

(c) For $j \neq k$, supp $\Theta_{\mu}^{j}=\operatorname{supp} W_{\mu}^{j}$ and supp $\Theta_{\mu}^{j} \cap \operatorname{supp} W_{\mu}^{k}=\emptyset$.

Remark 4.2 In particular notice that if we choose

$$
a=\frac{d-1}{p}, \quad b=\frac{d-1}{p^{\prime}}
$$

and we define the constant $M$ in the statement of Proposition 3.1 as

$$
M:=2 d \max \left\{\|\Phi\|_{L^{\infty}\left(\mathbb{R}^{d-1}\right)},\|\Phi\|_{L^{\infty}\left(\mathbb{R}^{d-1}\right)}^{2},\|\nabla \Phi\|_{L^{\infty}\left(\mathbb{R}^{d-1}\right)}\right\},
$$

then the following estimates hold:

$$
\sum_{j=1}^{d}\left\|\Theta_{\mu}^{j}\right\|_{L^{p}\left(\mathbb{T}^{d}\right)}, \sum_{j=1}^{d}\left\|W_{\mu}^{j}\right\|_{L^{p^{\prime}\left(\mathbb{T}^{d}\right)}}, \sum_{j=1}^{d}\left\|\Theta_{\mu}^{j} W_{\mu}^{j}\right\|_{L^{1}\left(\mathbb{T}^{d}\right)} \leq \frac{M}{2}
$$

and

$$
\left\|\Theta_{\mu}^{j}\right\|_{L^{1}\left(\mathbb{T}^{d}\right)},\left\|W_{\mu}^{j}\right\|_{L^{1}\left(\mathbb{T}^{d}\right)},\left\|W_{\mu}^{j}\right\|_{W^{1, \tilde{p}}} \leq M \mu^{-\gamma},
$$

where

$$
\gamma=\min \left\{\gamma_{1}, \gamma_{2}, \gamma_{3}\right\}>0
$$


and

$$
\begin{aligned}
& \gamma_{1}:=(d-1)\left(1-\frac{1}{p}\right)>0, \\
& \gamma_{2}:=(d-1)\left(1-\frac{1}{p^{\prime}}\right)>0, \\
& \gamma_{3}:=-1-(d-1)\left[\frac{1}{p^{\prime}}-\frac{1}{\tilde{p}}\right]=(d-1)\left[\frac{1}{p}+\frac{1}{\tilde{p}}-\left(1+\frac{1}{d-1}\right)\right]>0 .
\end{aligned}
$$

Notice that $\gamma_{3}>0$ by (12).

Proof of Proposition 4.1 Step 1 For each $j=1, \ldots, d$, we define the (non-periodic) Mikado density $\tilde{\Theta}_{\mu}^{j}: \mathbb{R}^{d} \rightarrow \mathbb{R}$

$$
\tilde{\Theta}_{\mu}^{j}\left(x_{1}, \ldots, x_{n}\right):=\mu^{a} \Phi_{\mu}\left(x_{1}, \ldots, x_{j-1}, x_{j+1}, \ldots, x_{d}\right)
$$

and the (non-periodic) Mikado field $\tilde{W}_{\mu}^{j}: \mathbb{R}^{d} \rightarrow \mathbb{R}^{d}$

$$
\tilde{W}_{\mu}^{j}\left(x_{1}, \ldots, x_{n}\right):=\mu^{b} \Phi_{\mu}\left(x_{1}, \ldots, x_{j-1}, x_{j+1}, \ldots, x_{d}\right) e_{j}
$$

Notice that for the non-periodic Mikado densities

$$
\begin{cases}\operatorname{div} \tilde{W}_{\mu}^{j} & =0 \\ \operatorname{div}\left(\tilde{\Theta}_{\mu}^{j} \tilde{W}_{\mu}^{j}\right) & =0 \\ f_{(0,1)^{d}} \tilde{\Theta}_{\mu}^{j}=f_{(0,1)^{d}} \tilde{W}_{\mu}^{j} & =0 \\ f_{(0,1)^{d}} \tilde{\Theta}_{\mu}^{j} \tilde{W}_{\mu}^{j} & =e_{j},\end{cases}
$$

where the last equality follows from (33). Moreover, from (32) we get

$$
\begin{aligned}
\left\|D^{k} \tilde{\Theta}_{\mu}^{j}\right\|_{L^{r}\left((0,1)^{d}\right)} & =\mu^{a+k-(d-1) / r}\left\|D^{k} \Phi\right\|_{L^{r}\left(\mathbb{R}^{d-1}\right)} \\
\left\|D^{k} \tilde{W}_{\mu}^{j}\right\|_{L^{r}\left((0,1)^{d}\right)} & =\mu^{b+k-(d-1) / r}\left\|D^{k} \Phi\right\|_{L^{r}\left(\mathbb{R}^{d-1}\right)}
\end{aligned}
$$

Step 2 We define $\Theta_{\mu}^{j}: \mathbb{T}^{d} \rightarrow \mathbb{R}$ and $W_{\mu}^{j}: \mathbb{T}^{d} \rightarrow \mathbb{R}^{d}$ as the 1-periodic extension of $\tilde{\Theta}_{\mu}^{j}, \tilde{W}_{\mu}^{j}$ respectively. Such periodic extensions are well defined, since supp $\Phi \subseteq$ $(0,1)^{d-1}$ and $\tilde{\Theta}_{\mu}^{j}, \tilde{W}_{\mu}^{j}$ do not depend on the $j$-th coordinate. Equations (34) and estimates (35) come from the corresponding equations (40) and estimates (41) for the non-periodic Mikado densities and fields.

Step 3 Finally notice that conditions (c) in the statement are not verified by $\Theta_{\mu}^{j}$ and $W_{\mu}^{j}$ defined in Step 2. However we can achieve (c), using that $\mu>2 d$ and redefining $\Theta_{\mu}^{j}, W_{\mu}^{j}$ after a suitable translation of the independent variable $x \in \mathbb{T}^{d}$ for each $j=1, \ldots, d$. 


\subsection{Definition of the Perturbations}

We are now in a position to define $\rho_{1}, u_{1}$. The constant $M$ has already been fixed in (36). Let thus $\eta, \delta, \sigma>0$ and $\left(\rho_{0}, u_{0}, R_{0}\right)$ be a smooth solution to the continuity-defect equation (25).

Let

$$
\begin{gathered}
\lambda \in \mathbb{N} \text { "oscillation", } \\
\mu>2 d \text { "concentration" }
\end{gathered}
$$

be two constant, which will be fixed in Sect. 6. Let $\psi \in C_{c}^{\infty}((0,1))$ such that $\psi \equiv 0$ on $[0, \sigma / 2] \cup[1-\sigma / 2,1], \psi \equiv 1$ on $[\sigma, 1-\sigma]$ and $|\psi| \leq 1$. We denote by $R_{0, j}$ the components of $R_{0}$, i.e.

$$
R_{0}(t, x):=\sum_{j=1}^{d} R_{0, j}(t, x) e_{j} .
$$

For $j=1, \ldots, d$, let $\chi_{j} \in C^{\infty}\left([0,1] \times \mathbb{T}^{d}\right)$ be such that

$$
\chi_{j}(t, x)= \begin{cases}0, & \text { if }\left|R_{0, j}(t, x)\right| \leq \delta /(4 d) \\ 1, & \text { if }\left|R_{0, j}(t, x)\right| \geq \delta /(2 d)\end{cases}
$$

and $\left|\chi_{j}\right| \leq 1$.

We set

$$
\rho_{1}:=\rho_{0}+\vartheta+\vartheta_{c}, \quad u_{1}:=u_{0}+w+w_{c}
$$

where $\vartheta, \vartheta_{c}, w, w_{c}$ are defined as follows. First of all, let $\Theta_{\mu}^{j}, W_{\mu}^{j}, j=1, \ldots, d$, be the Mikado densities and flows provided by Proposition 4.1, with $a, b$ chosen as in Remark 4.2. We set

$$
\begin{aligned}
\vartheta(t, x) & :=\eta \sum_{j=1}^{d} \psi(t) \chi_{j}(t, x) \operatorname{sign}\left(R_{0, j}(t, x)\right)\left|R_{0, j}(t, x)\right|^{1 / p} \Theta_{\mu}^{j}(\lambda x), \\
\vartheta_{c}(t) & :=-f_{\mathbb{T}^{d}} \vartheta(t, x) d x \\
w(t, x) & :=\eta^{-1} \sum_{j=1}^{d} \psi(t) \chi_{j}(t, x)\left|R_{0, j}(t, x)\right|^{1 / p^{\prime}} W_{\mu}^{j}(\lambda x) .
\end{aligned}
$$

We will also use the shorter notation

$$
\begin{aligned}
& \vartheta(t)=\eta \sum_{j=1}^{d} \psi(t) \chi_{j}(t) \operatorname{sign}\left(R_{0, j}(t)\right)\left|R_{0, j}(t)\right|^{1 / p}\left(\Theta_{\mu}^{j}\right)_{\lambda}, \\
& w(t)=\eta^{-1} \sum_{j=1}^{d} \psi(t) \chi_{j}(t)\left|R_{0, j}(t)\right|^{1 / p^{\prime}}\left(W_{\mu}^{j}\right)_{\lambda},
\end{aligned}
$$


where, coherent with (18),

$$
\left(\Theta_{\mu}^{j}\right)_{\lambda}(x)=\Theta_{\mu}^{j}(\lambda x), \quad\left(W_{\mu}^{j}\right)_{\lambda}(x)=W_{\mu}^{j}(\lambda x) .
$$

Notice that $\vartheta$ and $w$ are smooth functions, thanks to the cutoffs $\chi_{j}$. Notice also that $\vartheta+\vartheta_{c}$ has zero mean value in $\mathbb{T}^{d}$ at each time $t$. To define $w_{c}$, notice first that

$$
-\operatorname{div} w(t)=-\eta^{-1} \sum_{j=1}^{d} \nabla\left(\psi(t) \chi_{j}(t)\left|R_{0, j}(t)\right|^{1 / p}\right) \cdot\left(W_{\mu}^{j}\right)_{\lambda}
$$

is sum of terms of the form $f \cdot g_{\lambda}$, each term has zero mean value (being a divergence) and the fast oscillatory term $W_{\mu}^{j}$ has zero mean value as well. We can therefore apply Lemma 2.3 and define

$$
w_{c}(t):=-\eta^{-1} \sum_{j=1}^{d} \mathcal{R}\left(\nabla\left(\psi(t) \chi_{j}(t)\left|R_{0, j}(t)\right|^{1 / p}\right) \cdot\left(W_{\mu}^{j}\right)_{\lambda}\right) .
$$

Then $\operatorname{div}\left(w+w_{c}\right)=0$ and thus

$$
\operatorname{div} u_{1}=\operatorname{div} u_{0}+\operatorname{div}\left(w+w_{c}\right)=0 .
$$

Moreover, by Remark 2.5, $w_{c}$ is smooth in $(t, x)$.

\subsection{Estimates on the Perturbation}

In this section we provide some estimates on $\vartheta, \vartheta_{c}, w, w_{c}$.

Lemma $4.3\left(L^{p}\right.$-norm of $\left.\vartheta\right)$ For every time $t \in[0,1]$,

$$
\|\vartheta(t)\|_{L^{p}\left(\mathbb{T}^{d}\right)} \leq \frac{M}{2} \eta\left\|R_{0}(t)\right\|_{L^{1}\left(\mathbb{T}^{d}\right)}^{1 / p}+\frac{C\left(\eta, \delta,\left\|R_{0}(t)\right\|_{C^{1}\left(\mathbb{T}^{d}\right)}\right)}{\lambda^{1 / p}} .
$$

Proof The perturbation $\vartheta$ is the sum of functions of the form $f g_{\lambda}$. Therefore we can apply the improved Hölder inequality, Lemma 2.1, to get

$$
\begin{aligned}
\|\vartheta(t)\|_{L^{p}} \leq & \eta \sum_{j=1}^{d}\left\|\psi(t) \chi_{j}(t) \operatorname{sign}\left(R_{0, j}(t)\right)\left|R_{0, j}(t)\right|^{1 / p}\right\|_{L^{p}}\left\|\Theta_{\mu}^{j}\right\|_{L^{p}} \\
& +\frac{C_{p}}{\lambda^{1 / p}}\left\|\psi(t) \chi_{j}(t) \operatorname{sign}\left(R_{0, j}(t)\right)\left|R_{0, j}(t)\right|^{1 / p}\right\|_{C^{1}\left(\mathbb{T}^{d}\right)}\left\|\Theta_{\mu}^{j}\right\|_{L^{p}}
\end{aligned}
$$

Notice now that

$$
\left\|\psi(t) \chi_{j}(t) \operatorname{sign}\left(R_{0, j}(t)\right)\left|R_{0, j}(t)\right|^{1 / p}\right\|_{L^{p}} \leq\left\|\left|R_{0, j}(t)\right|^{1 / p}\right\|_{L^{p}} \leq\left\|R_{0}(t)\right\|_{L^{1}}^{1 / p}
$$


and, recalling the definition of the cutoff $\chi_{j}$ in Sect. 4.2,

$$
\left\|\psi(t) \chi_{j}(t) \operatorname{sign}\left(R_{0, j}(t)\right)\left|R_{0, j}(t)\right|^{1 / p}\right\|_{C^{1}\left(\mathbb{T}^{d}\right)} \leq C\left(\delta,\left\|R_{0}(t)\right\|_{C^{1}\left(\mathbb{T}^{d}\right)}\right) .
$$

Therefore, using the bounds on $\left\|\Theta_{\mu}^{j}\right\|_{L^{p}}$ provided in (37), we get

$$
\|\vartheta(t)\|_{L^{p}} \leq \frac{M}{2} \eta\left\|R_{0}(t)\right\|_{L^{1}}^{1 / p}+\frac{C\left(\eta, \delta,\left\|R_{0}(t)\right\|_{C^{1}}\right)}{\lambda^{1 / p}} .
$$

Lemma 4.4 (Estimate on $\vartheta_{c}$ ) It holds

$$
\left|\vartheta_{c}(t)\right| \leq \frac{C\left(\eta,\left\|R_{0}(t)\right\|_{C^{1}\left(\mathbb{T}^{d}\right)}\right)}{\lambda}
$$

Proof We use Lemma 2.6:

$$
\begin{aligned}
\left|\vartheta_{c}(t)\right| & \leq \eta \sum_{j=1}^{d} \frac{\sqrt{d}\left\|R_{0}(t)\right\|_{C^{1}\left(\mathbb{T}^{d}\right)}\left\|\Theta_{\mu}^{j}\right\|_{L^{1}}}{\lambda} \\
& \leq \frac{C\left(\eta,\left\|R_{0}(t)\right\|_{C^{1}\left(\mathbb{T}^{d}\right)}\right)}{\lambda}
\end{aligned}
$$

Lemma $4.5\left(L^{p^{\prime}}\right.$ norm of $\left.w\right)$ For every time $t \in[0,1]$,

$$
\|w(t)\|_{L^{p^{\prime}\left(\mathbb{T}^{d}\right)}} \leq \frac{M}{2 \eta}\left\|R_{0}(t)\right\|_{L^{1}\left(\mathbb{T}^{d}\right)}^{1 / p^{\prime}}+\frac{C\left(\eta, \delta,\left\|R_{0}(t)\right\|_{C^{1}\left(\mathbb{T}^{d}\right)}\right)}{\lambda^{1 / p^{\prime}}} .
$$

Proof The proof is completely analogous to the proof of Lemma 4.3, with $\eta^{-1}$ instead of $\eta$ and $\left\|W_{\mu}^{j}\right\|_{L^{p^{\prime}}}$ instead of $\left\|\Theta_{\mu}^{j}\right\|_{L^{p}}$, and thus it is omitted.

Lemma $4.6\left(W^{1, \tilde{p}}\right.$ norm of $\left.w\right)$ For every time $t \in[0,1]$,

$$
\|w(t)\|_{W^{1, \tilde{p}}\left(\mathbb{T}^{d}\right)} \leq C\left(\eta,\left\|R_{0}\right\|_{C^{1}}\right) \lambda \mu^{-\gamma}
$$

Proof We have

$$
D w(t, x)=\eta^{-1} \psi(t) \sum_{j=1}^{d} W_{\mu}^{j}(\lambda x) \otimes D\left(\chi_{j}\left|R_{0, j}\right|^{1 / p^{\prime}}\right)+\lambda \chi_{j}\left|R_{0, j}\right|^{1 / p^{\prime}} D W_{\mu}^{j}(\lambda x),
$$

from which we get the pointwise estimate

$$
|D w(t, x)| \leq C\left(\eta, \delta,\left\|R_{0}\right\|_{C^{1}}\right) \sum_{j=1}^{d}\left(\left|W_{\mu}^{j}(\lambda x)\right|+\lambda\left|D W_{\mu}^{j}(\lambda x)\right|\right) .
$$


We can take now the $L^{\tilde{p}}$ norm of $D w(t)$ and use (38) to get

$$
\begin{aligned}
\|D w(t)\|_{L^{\tilde{p}}} & \leq C\left(\eta, \delta,\left\|R_{0}\right\|_{C^{1}}\right) \sum_{j=1}^{d}\left(\left\|W_{\mu}^{j}\right\|_{L^{\tilde{p}}}+\lambda\left\|D W_{\mu}^{j}\right\|_{L^{\tilde{p}}}\right) \\
& \leq C\left(\eta, \delta,\left\|R_{0}\right\|_{C^{1}}\right) \lambda \mu^{-\gamma} .
\end{aligned}
$$

A similar (and even easier) computation holds for $\|w(t)\|_{L^{\tilde{p}}}$, thus concluding the proof of the lemma.

Lemma $4.7\left(L^{p^{\prime}}\right.$ norm of $\left.w_{c}\right)$ For every time $t \in[0,1]$,

$$
\left\|w_{c}(t)\right\|_{L^{p^{\prime}}\left(\mathbb{T}^{d}\right)} \leq \frac{C\left(\eta, \delta,\left\|R_{0}\right\|_{C^{2}}\right)}{\lambda} .
$$

Proof The corrector $w_{c}$ is defined in (43) using the antidivergence operator of Lemma 2.3. We can thus use the bounds given by that lemma, with $k=0$, to get

$$
\begin{aligned}
\left\|w_{c}(t)\right\|_{L^{p^{\prime}}} & \leq \eta^{-1} \sum_{j=1}^{d} \frac{C_{0, p^{\prime}}}{\lambda}\left\|\nabla\left(\psi(t) \chi_{j}(t)\left|R_{0, j}(t)\right|^{1 / p^{\prime}}\right)\right\|_{C^{1}\left(\mathbb{T}^{d}\right)}\left\|W_{\mu}^{j}\right\|_{L^{p^{\prime}}} \\
& \leq \frac{C\left(\eta, \delta,\left\|R_{0}\right\|_{C^{2}}\right)}{\lambda} \sum_{j=1}^{d}\left\|W_{\mu}^{j}\right\|_{L^{p^{\prime}}} \\
\text { (by (37)) } & \leq \frac{C\left(\eta, \delta,\left\|R_{0}\right\|_{\left.C^{2}\right)}\right.}{\lambda} .
\end{aligned}
$$

Lemma $4.8\left(W^{1, \tilde{p}}\right.$ norm of $\left.w_{c}\right)$ For every time $t \in[0,1]$,

$$
\left\|w_{c}(t)\right\|_{W^{1, \tilde{p}}\left(\mathbb{T}^{d}\right)} \leq C\left(\eta, \delta,\left\|R_{0}\right\|_{C^{3}}\right) \mu^{-\gamma} .
$$

Proof We estimate only $\left\|D w_{c}(t)\right\|_{L \tilde{p}}$, the estimate for $\left\|w_{c}(t)\right\|_{L \tilde{p}}$ is analogous and even easier. We use once again the bounds provided by Lemma 2.3 with $k=1$ :

$$
\begin{aligned}
\left\|D w_{c}(t)\right\|_{L^{\tilde{p}}} & \leq \eta^{-1} C_{1, \tilde{p}} \sum_{j=1}^{d}\left\|\nabla\left(\psi(t) \chi_{j}(t)\left|R_{0, j}(t)\right|^{1 / p}\right)\right\|_{C^{2}\left(\mathbb{T}^{d}\right)}\left\|W_{\mu}^{j}\right\|_{W^{1, \tilde{p}}} \\
(\operatorname{by~}(38)) & \leq C\left(\eta, \delta,\left\|R_{0}\right\|_{C^{3}}\right) \mu^{-\gamma} .
\end{aligned}
$$

\section{The New Defect Field}

In this section we continue the proof of Proposition 3.1, defining the new defect field $R_{1}$ and proving some estimates on it. 


\subsection{Definition of the New Defect Field}

We want to define $R_{1}$ so that

$$
-\operatorname{div} R_{1}=\partial_{t} \rho_{1}+\operatorname{div}\left(\rho_{1} u_{1}\right) .
$$

Let us compute

$$
\begin{aligned}
& \partial_{t} \rho_{1}+\operatorname{div}\left(\rho_{1} u_{1}\right)= \operatorname{div}\left(\vartheta w-R_{0}\right) \\
&+ \partial_{t}\left(\vartheta+\vartheta_{c}\right)+\operatorname{div}\left(\vartheta u_{0}+\rho_{0} w\right) \\
&+\operatorname{div}\left(\rho_{0} w_{c}+\vartheta_{c} u_{0}+\vartheta w_{c}+\vartheta_{c} w+\vartheta_{c} w_{c}\right) \\
&=\operatorname{div}\left[\left(\vartheta w-R_{0}\right)\right. \\
& \quad+\left(\nabla \Delta^{-1} \partial_{t}\left(\vartheta+\vartheta_{c}\right)+\vartheta_{0}+\rho_{0} w\right) \\
&\left.\quad+\left(\rho_{0} w_{c}+\vartheta_{c} u_{0}+\vartheta w_{c}+\vartheta_{c} w+\vartheta_{c} w_{c}\right)\right] \\
&=\operatorname{div}\left[\left(\vartheta w-R_{0}\right)+R^{\text {linear }}+R^{\text {corr }}\right]
\end{aligned}
$$

where we put

$$
\begin{aligned}
R^{\text {linear }} & :=\nabla \Delta^{-1} \partial_{t}\left(\vartheta+\vartheta_{c}\right)+\vartheta u_{0}+\rho_{0} w \\
R^{\text {corr }} & :=\rho_{0} w_{c}+\vartheta_{c} u_{0}+\vartheta w_{c}+\vartheta_{c} w+\vartheta_{c} w_{c} .
\end{aligned}
$$

Note that we can apply the antidivergence operator $\nabla \Delta^{-1}$ to $\partial_{t}\left(\vartheta+\vartheta_{c}\right)$, since it has zero mean value. Let us now consider the term $\vartheta w-R_{0}$. Recall from Proposition 4.1, that, for $j \neq k$, supp $\Theta_{\mu}^{j} \cap \operatorname{supp} W_{\mu}^{k}=\emptyset$. Coherent with (18), we use the notation

$$
\left(\Theta_{\mu}^{j} W_{\mu}^{j}\right)_{\lambda}(x)=\Theta_{\mu}^{j}(\lambda x) W_{\mu}^{j}(\lambda x) .
$$

We have

$$
\begin{aligned}
\vartheta(t) w(t)-R_{0}(t)= & \sum_{j=1}^{d} \psi^{2}(t) \chi_{j}^{2}(t) R_{0, j}(t)\left(\Theta_{\mu}^{j} W_{\mu}^{j}\right)_{\lambda}-R_{0}(t) \\
= & \sum_{j=1}^{d} \psi^{2}(t) \chi_{j}^{2}(t) R_{0, j}(t)\left[\left(\Theta_{\mu}^{j} W_{\mu}^{j}\right)_{\lambda}-e_{j}\right] \\
& +\psi^{2}(t) \sum_{j=1}^{d}\left[\chi_{j}^{2}(t)-1\right] R_{0, j}(t) e_{j} \\
& +\left[\psi^{2}(t)-1\right] R_{0}(t) \\
= & \sum_{j=1}^{d} \psi^{2}(t) \chi_{j}^{2}(t) R_{0, j}(t)\left[\left(\Theta_{\mu}^{j} W_{\mu}^{j}\right)_{\lambda}-e_{j}\right] \\
& +R^{\chi}(t)+R^{\psi}(t),
\end{aligned}
$$


where we put

$$
\begin{aligned}
& R^{\chi}(t):=\psi^{2}(t) \sum_{j=1}^{d}\left[\chi_{j}^{2}(t)-1\right] R_{0, j}(t) e_{j}, \\
& R^{\psi}(t):=\left[\psi^{2}(t)-1\right] R_{0}(t) .
\end{aligned}
$$

Thus, using again Proposition 4.1, and in particular the fact that $\operatorname{div}\left(\Theta_{\mu}^{j} W_{\mu}^{j}\right)=0$, we get

$$
\begin{aligned}
\operatorname{div}\left(\vartheta(t) w(t)-R_{0}(t)\right)= & \sum_{j=1}^{d} \nabla\left(\psi^{2}(t) \chi_{j}^{2}(t) R_{0, j}(t)\right) \cdot\left[\left(\Theta_{\mu}^{j} W_{\mu}^{j}\right)_{\lambda}-e_{j}\right] \\
& +\operatorname{div}\left(R^{\chi}+R^{\psi}\right) .
\end{aligned}
$$

Each term in the summation over $j$ has the form $f \cdot g_{\lambda}$ and it has zero mean value, being a divergence. Moreover, again by Proposition 4.1,

$$
f_{\mathbb{T}^{d}}\left(\Theta_{\mu}^{j} W_{\mu}^{j}\right)_{\lambda} d x=f_{\mathbb{T}^{d}} \Theta_{\mu}^{j} W_{\mu}^{j} d x=e_{j}
$$

Therefore we can apply Lemma 2.3 and define

$$
R^{\text {quadr }}(t):=\sum_{j=1}^{d} \mathcal{R}\left(\nabla\left(\psi^{2}(t) \chi_{j}^{2}(t) R_{0, j}(t)\right) \cdot\left[\left(\Theta_{\mu}^{j} W_{\mu}^{j}\right)_{\lambda}-e_{j}\right]\right)
$$

By Remark 2.5, $R^{\text {quadr }}$ is smooth in $(t, x)$. Summarizing, from (44) and (47) we get

$$
\partial_{t} \rho_{1}+\operatorname{div}\left(\rho_{1} u_{1}\right)=\operatorname{div}\left[R^{\text {quadr }}+R^{\chi}+R^{\psi}+R^{\text {linear }}+R^{\text {corr }}\right] .
$$

We thus define

$$
-R_{1}:=R^{\text {quadr }}+R^{\chi}+R^{\psi}+R^{\text {linear }}+R^{\text {corr }} .
$$

Aim of the next section will be to get an estimate in $L^{1}$ for $R_{1}(t)$, by estimating separately each term in (49).

\subsection{Estimates on the Defect Field}

We now prove some estimates on the different terms which define $R_{1}$.

Lemma 5.1 (Estimate on $R^{\text {quadr }}$ ) For every $t \in[0,1]$,

$$
\left\|R^{\text {quadr }}(t)\right\|_{L^{1}\left(\mathbb{T}^{d}\right)} \leq \frac{C\left(\delta,\left\|R_{0}\right\|_{C^{2}}\right)}{\lambda} .
$$


Proof $R^{\text {quadr }}$ is defined in (48) using Lemma 2.3. Observe first that

$$
\left\|\nabla\left(\psi^{2}(t) \chi_{j}^{2}(t) R_{0, j}(t)\right)\right\|_{C^{1}\left(\mathbb{T}^{d}\right)} \leq C\left(\delta,\left\|R_{0}\right\|_{C^{2}}\right) .
$$

Applying the bounds provided by Lemma 2.3, with $k=0$, and (37) we get

$$
\begin{aligned}
& \left\|R^{\text {quadr }}(t)\right\|_{L^{1}\left(\mathbb{T}^{d}\right)} \\
& \quad \leq \sum_{j=1}^{d} \frac{C_{0,1}}{\lambda}\left\|\nabla\left(\psi^{2}(t) \chi_{j}^{2}(t) R_{0, j}(t)\right)\right\|_{C^{1}}\left\|\Theta_{\mu}^{j} W_{\mu}^{j}-e_{j}\right\|_{L^{1}\left(\mathbb{T}^{d}\right)} \\
& \quad \leq \sum_{j=1}^{d} \frac{C\left(\delta,\left\|R_{0}\right\|_{C^{2}}\right)}{\lambda}
\end{aligned}
$$

Lemma 5.2 (Estimate on $\left.R^{\chi}\right)$ For every $t \in[0,1]$

$$
\left\|R^{\chi}(t)\right\|_{L^{1}\left(\mathbb{T}^{d}\right)} \leq \frac{\delta}{2}
$$

Proof Notice that $\chi_{j}(t, x)=1$ if $\left|R_{0, j}(t, x)\right| \geq \delta /(2 d)$. Therefore $R^{\chi}(t, x) \neq 0$ only when $\left|R_{0, j}(t, x)\right| \leq \delta /(2 d)$. We thus have the pointwise estimate

$$
\left|R^{\chi}(t, x)\right| \leq \sum_{j=1}^{d}\left|\chi_{j}(t, x)^{2}-1\right|\left|R_{0, j}(t, x)\right| \leq \frac{\delta}{2} .
$$

from which the conclusion easily follows.

Lemma 5.3 (Estimate on $R^{\psi}$ ) It holds

$$
\left\|R^{\psi}(t)\right\|_{L^{1}\left(\mathbb{T}^{d}\right)} \leq \begin{cases}0, & t \in I_{\sigma}, \\ \left\|R_{0}(t)\right\|_{L^{1}\left(\mathbb{T}^{d}\right)}, & t \in[0,1] \backslash I_{\sigma}\end{cases}
$$

Proof The proof follows immediately from the definition of $R^{\psi}$ in (46) and the definition of the cutoff $\psi$.

Lemma 5.4 (Estimate on $R^{\text {linear }}$ ) For every $t \in[0,1]$

$$
\left\|R^{\text {linear }}(t)\right\|_{L^{1}\left(\mathbb{T}^{d}\right)} \leq C\left(\eta, \delta, \sigma,\left\|\rho_{0}\right\|_{C^{0}},\left\|u_{0}\right\|_{C^{0}},\left\|R_{0}\right\|_{C^{0}}\right) \mu^{-\gamma} .
$$

Proof At each time $t \in[0,1]$,

$$
\begin{aligned}
& \left\|R^{\text {linear }}(t)\right\|_{L^{1}\left(\mathbb{T}^{d}\right)} \\
& \quad \leq\left\|\nabla \Delta^{-1} \partial_{t}\left(\vartheta(t)+\vartheta_{c}(t)\right)\right\|_{L^{1}\left(\mathbb{T}^{d}\right)}+\left\|\vartheta(t) u_{0}(t)\right\|_{L^{1}\left(\mathbb{T}^{d}\right)}+\left\|\rho_{0}(t) w(t)\right\|_{L^{1}\left(\mathbb{T}^{d}\right)} \\
& \quad \leq\left\|\partial_{t} \vartheta(t)\right\|_{L^{1}\left(\mathbb{T}^{d}\right)}+\left|\vartheta_{c}^{\prime}(t)\right|+\left\|\vartheta(t) u_{0}(t)\right\|_{L^{1}\left(\mathbb{T}^{d}\right)}+\left\|\rho_{0}(t) w(t)\right\|_{L^{1}\left(\mathbb{T}^{d}\right)},
\end{aligned}
$$


where the first term was estimated using Lemma 2.2. We now separately estimate each term in the last sum.

1. Estimate on $\left\|\partial_{t} \vartheta(t)\right\|_{L^{1}}$. We have

$$
\partial_{t} \vartheta(t)=\eta \sum_{j=1}^{d} \partial_{t}\left(\psi(t) \chi_{j}(t, x) \operatorname{sign}\left(R_{0, j}(t, x)\right)\left|R_{0, j}(t, x)\right|^{1 / p}\right) \Theta_{\mu}^{j}(\lambda x)
$$

from which we get the pointwise estimate

$$
\left|\partial_{t} \vartheta(t)\right| \leq C\left(\eta, \delta, \sigma,\left\|R_{0}\right\|_{C^{1}}\right) \sum_{j=1}^{d}\left|\Theta_{\mu}^{j}(\lambda x)\right|
$$

Using (38), we deduce

$$
\left\|\partial_{t} \vartheta(t)\right\|_{L^{1}} \leq C\left(\eta, \delta, \sigma,\left\|R_{0}\right\|_{C^{1}}\right) \mu^{-\gamma} .
$$

2. Estimate on $\left|\vartheta_{c}^{\prime}(t)\right|$. We have

$$
\left|\vartheta_{c}^{\prime}(t)\right| \leq\left\|\partial_{t} \vartheta(t)\right\|_{L^{1}} \leq C\left(\eta, \delta, \sigma,\left\|R_{0}\right\|_{C^{1}}\right) \mu^{-\gamma}
$$

3. Estimate on $\left\|\vartheta(t) u_{0}(t)\right\|_{L^{1}}$. We now use the classical Hölder inequality to estimate

$$
\begin{aligned}
\left\|\vartheta(t) u_{0}(t)\right\|_{L^{1}} & \leq\left\|u_{0}\right\|_{C^{0}}\|\vartheta(t)\|_{L^{1}} \\
& \leq \eta\left\|u_{0}\right\|_{C^{0}} \sum_{j=1}^{d}\left\|\left|R_{0, j}\right|^{1 / p}\right\|_{C^{0}}\left\|\Theta_{\mu}^{j}\right\|_{L^{1}} \\
(\text { by }(38)) & \leq C\left(\eta,\left\|u_{0}\right\|_{C^{0}},\left\|R_{0}\right\|_{C^{0}}\right) \mu^{-\gamma} .
\end{aligned}
$$

4. Estimate on $\left\|\rho_{0}(t) w(t)\right\|_{L^{1}}$. Similarly, again using the classical Hölder inequality,

$$
\begin{aligned}
\left\|\rho_{0}(t) w(t)\right\|_{L^{1}} & \leq\left\|\rho_{0}\right\|_{C^{0}}\|w(t)\|_{L^{1}} \\
& \leq \eta^{-1}\left\|\rho_{0}\right\|_{C^{0}} \sum_{j=1}^{d}\left\|\left|R_{0, j}\right|^{1 / p^{\prime}}\right\|_{C^{0}}\left\|W_{\mu}^{j}\right\|_{L^{1}} \\
\quad(\text { by }(38)) & \leq C\left(\eta,\left\|\rho_{0}\right\|_{C^{0}},\left\|R_{0}\right\|_{C^{0}}\right) \mu^{-\gamma} .
\end{aligned}
$$

Lemma 5.5 (Estimate on $R^{\text {corr }}$ ) For every $t \in[0,1]$,

$$
\left\|R^{\mathrm{corr}}(t)\right\|_{L^{1}\left(\mathbb{T}^{d}\right)} \leq \frac{C\left(\eta, \delta,\left\|\rho_{0}\right\|_{C^{0}},\left\|u_{0}\right\|_{C^{0}},\left\|R_{0}\right\|_{C^{2}}\right)}{\lambda}
$$


Proof We estimate separately each term in the definition (45) of $R^{\text {corr }}$.

1. Estimate on $\rho_{0} w_{c}$. By the classical Hölder inequality,

$$
\begin{aligned}
\left\|\rho_{0}(t) w_{c}(t)\right\|_{L^{1}} & \leq\left\|\rho_{0}\right\|_{C^{0}}\left\|w_{c}(t)\right\|_{L^{1}} \\
& \leq\left\|\rho_{0}\right\|_{C^{0}}\left\|w_{c}(t)\right\|_{L^{p^{\prime}}} \\
\text { (by Lemma 4.7) } & \leq \frac{C\left(\eta, \delta,\left\|\rho_{0}\right\|_{C^{0}},\left\|R_{0}\right\|_{C^{2}}\right)}{\lambda} .
\end{aligned}
$$

2. Estimate on $\vartheta_{c} u_{0}$. We use Lemma 4.4:

$$
\left\|\vartheta_{c}(t) u_{0}(t)\right\|_{L^{1}} \leq\left|\vartheta_{c}(t)\right|\left\|u_{0}\right\|_{C^{0}} \leq \frac{C\left(\eta,\left\|u_{0}\right\|_{C^{0}},\left\|R_{0}\right\|_{C^{2}}\right)}{\lambda} .
$$

3. Estimate on $\vartheta w_{c}$. We use Lemmas 4.3 and 4.7:

$$
\left\|\vartheta(t) w_{c}(t)\right\|_{L^{1}} \leq\|\vartheta(t)\|_{L^{p}}\left\|w_{c}(t)\right\|_{L^{p^{\prime}}} \leq \frac{C\left(\eta, \delta,\left\|R_{0}\right\|_{C^{2}}\right)}{\lambda}
$$

4. Estimate on $\vartheta_{c} w$. We use Lemmas 4.4 and 4.5:

$$
\begin{aligned}
\left\|\vartheta_{c}(t) w(t)\right\|_{L^{1}} & \leq\left|\vartheta_{c}(t)\right|\|w(t)\|_{L^{1}} \\
& \leq\left|\vartheta_{c}(t)\right|\|w(t)\|_{L^{p^{\prime}}} \\
& \leq \frac{C\left(\eta, \delta,\left\|R_{0}\right\|_{C^{2}}\right)}{\lambda} .
\end{aligned}
$$

5. Estimate on $\vartheta_{c} w_{c}$. We use Lemmas 4.4 and 4.7:

$$
\begin{aligned}
\left\|\vartheta_{c}(t) w_{c}(t)\right\|_{L^{1}} & \leq\left|\vartheta_{c}(t)\right|\left\|w_{c}(t)\right\|_{L^{1}} \\
& \leq\left|\vartheta_{c}(t)\right|\left\|w_{c}(t)\right\|_{L^{p^{\prime}}} \\
& \leq \frac{C\left(\eta, \delta,\left\|R_{0}\right\|_{C^{2}}\right)}{\lambda^{2}} .
\end{aligned}
$$

Remark 5.6 In estimating $R^{\text {corr }}$ the only term where we really need the fast oscillation $\lambda$ is the estimate on $\vartheta w_{c}$. All the other terms could be alternatively estimated using the concentration parameter $\mu$, since, by (38), $\left|\vartheta_{c}(t)\right|,\left\|w_{c}(t)\right\|_{L^{1}},\|w(t)\|_{L^{1}} \leq$ const. $\mu^{-\gamma}$. In this way we would obtained the less refined estimate

$$
\left\|R^{\mathrm{corr}}(t)\right\|_{L^{1}} \leq \frac{C}{\lambda}+\frac{C}{\mu^{\gamma}}
$$

which is however enough to prove Proposition 3.1. 


\section{Proof of Proposition 3.1}

In this section we conclude the proof of Proposition 3.1, proving estimates (26a)-(26d). We will choose

$$
\mu=\lambda^{c}
$$

for a suitable $c>1$ and $\lambda$ sufficiently large.

1. Estimate (26a). We have

$$
\begin{aligned}
\left\|\rho_{1}(t)-\rho_{0}(t)\right\|_{L^{p}} \leq & \left\|\vartheta_{0}(t)\right\|_{L^{p}}+\left|\vartheta_{c}(t)\right| \\
(\text { Lemmas 4.3 and 4.4) } \leq & \frac{M}{2} \eta\left\|R_{0}(t)\right\|_{L^{1}}^{1 / p}+\frac{C\left(\eta, \delta,\left\|R_{0}(t)\right\|_{C^{1}}\right)}{\lambda^{1 / p}} \\
& +\frac{C\left(\eta,\left\|R_{0}(t)\right\|_{C^{1}}\right)}{\lambda} \\
\leq & M \eta\left\|R_{0}(t)\right\|_{L^{1}}^{1 / p}
\end{aligned}
$$

if the constant $\lambda$ is chosen large enough. Notice also that, if $t \in[0,1] \backslash I_{\sigma / 2}$, then $\vartheta(t) \equiv 0$ and $\vartheta_{c}(t)=0$, thanks to the cutoff $\psi$ in (42). Therefore (26a) is proven.

2. Estimate (26b). The estimate uses Lemmas 4.5 and 4.7 and it is completely similar to what we just did for (26a).

4. Estimate (26c). By Lemma 4.6,

$$
\|w(t)\|_{W^{1, \tilde{p}}} \leq C\left(\eta,\left\|R_{0}\right\|_{C^{1}}\right) \lambda \mu^{-\gamma} \leq \delta
$$

if $\mu$ is chosen of the form $\mu=\lambda^{c}$ with $c>1 / \gamma$ and $\lambda$ is chosen large enough.

4. Estimate (26d). Recall the definition of $R_{1}$ in (49). Using Lemmas 5.1, 5.2, 5.3, 5.4, 5.5 , for $t \in I_{\sigma}$, we get

$$
\begin{aligned}
\left\|R_{1}(t)\right\|_{L^{1}} & \leq \frac{\delta}{2}+C\left(\eta, \delta, \sigma,\left\|\rho_{0}\right\|_{C^{0}},\left\|u_{0}\right\|_{C^{0}},\left\|R_{0}\right\|_{C^{2}}\right)\left(\frac{1}{\lambda}+\frac{1}{\mu^{\gamma}}\right) \\
& \leq \frac{\delta}{2}+C\left(\eta, \delta, \sigma,\left\|\rho_{0}\right\|_{C^{0}},\left\|u_{0}\right\|_{C^{0}},\left\|R_{0}\right\|_{C^{2}}\right)\left(\frac{1}{\lambda}+\frac{1}{\lambda^{c \gamma}}\right) \\
& \leq \delta
\end{aligned}
$$

provided $\lambda$ is chosen large enough. Similarly, for $t \in I_{\sigma / 2} \backslash I_{\sigma}$, we have

$$
\begin{aligned}
& \left\|R_{1}(t)\right\|_{L^{1}} \\
& \leq\left\|R_{0}(t)\right\|_{L^{1}}+\frac{\delta}{2}+C\left(\eta, \delta, \sigma,\left\|\rho_{0}\right\|_{C^{0}},\left\|u_{0}\right\|_{C^{0}},\left\|R_{0}\right\|_{C^{2}}\right)\left(\frac{1}{\lambda}+\frac{1}{\mu^{\gamma}}\right) \\
& \leq\left\|R_{0}(t)\right\|_{L^{1}}+\frac{\delta}{2}+C\left(\eta, \delta, \sigma,\left\|\rho_{0}\right\|_{C^{0}},\left\|u_{0}\right\|_{C^{0}},\left\|R_{0}\right\|_{C^{2}}\right)\left(\frac{1}{\lambda}+\frac{1}{\lambda^{c \gamma}}\right) \\
& \leq\left\|R_{0}(t)\right\|_{L^{1}}+\delta
\end{aligned}
$$


if $\lambda$ is chosen large enough. Finally, for $t \in[0,1] \backslash I_{\sigma / 2}$, the cutoff function $\psi(t) \equiv 0$, and thus $\vartheta(t)=\vartheta_{c}(t)=w(t)=w_{c}(t)=0$. Therefore $R_{1}(t)=R_{0}(t)$.

\section{Sketch of the Proofs of Theorems 1.6, 1.9, 1.10}

Theorems $1.6,1.9,1.10$ can be proven in a very similar way to Theorem 1.2 and thus we present just a sketch of their proofs.

The proof of Theorem 1.2 follows from Proposition 3.1: similarly, for each one of Theorems 1.6, 1.9, 1.10 there is a corresponding main proposition, from which the proof the theorem follows.

\subsection{Sketch of the proof of Theorem 1.9}

The proof of Theorem 1.9 follows from the next proposition, in a very similar way as Theorem 1.2 follows from Proposition 3.1. Let us consider the equation

$$
\left\{\begin{array}{l}
\partial_{t} \rho+\operatorname{div}(\rho u)-\Delta \rho=-\operatorname{div} R \\
\operatorname{div} u=0
\end{array}\right.
$$

Proposition 7.1 Proposition 3.1 holds with (50) instead of (25).

Sketch of the proof of Proposition 7.1 Exactly as in the proof of Proposition 3.1, we define the Mikado densities and fields as in Proposition 4.1 and we choose the exponents $a, b$ as in Remark 4.2. We observe that, in addition to (37), (38), it also holds

$$
\left\|\nabla \Theta_{\mu}^{j}\right\|_{L^{1}} \leq M \mu^{-\gamma_{4}} \leq M \mu^{-\gamma}
$$

for

$$
\gamma=\min \left\{\gamma_{1}, \gamma_{2}, \gamma_{3}, \gamma_{4}\right\}>0
$$

where $\gamma_{1}, \gamma_{2}, \gamma_{3}$ were defined in Remark 4.2 and

$$
\gamma_{4}:=\frac{d-1}{p^{\prime}}-1>0,
$$

because of the second condition in (15). Then the perturbations $\vartheta, w, \vartheta_{c}, w_{c}$ can be defined as in Sect. 4.2 and the estimates in Sect. 4.3 continue to hold. In the definition of the new defect field in Sect. 5 we want to define $R_{1}$ so that

$$
-\operatorname{div} R_{1}=\partial_{t} \rho_{1}+\operatorname{div}\left(\rho_{1} u_{1}\right)-\Delta \rho_{1},
$$

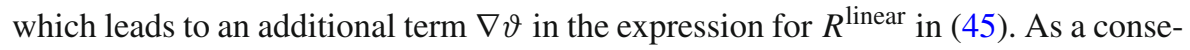
quence the only estimate which changes is Lemma 5.4. From (51) and the expression for $\vartheta$ in (42) we easily obtain

$$
\|\nabla \theta\|_{L^{1}\left(\mathbb{T}^{d}\right)} \leq C\left(\eta, \delta,\left\|R_{0}\right\|_{C^{1}}\right) \lambda \mu^{-\gamma} .
$$


Since we choose $\mu=\lambda^{c}$ with $c>1 / \gamma$ in Sect. 6 , the final estimates for $\left\|R_{1}(t)\right\|_{L^{1}}$ continue to hold. This concludes the proof of proposition (and thence also the proof of Theorem 1.9).

\subsection{Sketch of the proof of Theorem 1.6}

Also for Theorem 1.6 there is a main proposition, analog to Proposition 3.1.

Proposition 7.2 There exists a constant $M>0$ and an exponent $s \in(1, \infty)$ such that the following holds. Let $\eta, \delta, \sigma>0$ and let $\left(\rho_{0}, u_{0}, R_{0}\right)$ be a smooth solution of the continuity-defect equation (25). Then there exists another smooth solution $\left(\rho_{1}, u_{1}, R_{1}\right)$ of (25) such that

$$
\begin{aligned}
& \left\|\rho_{1}(t)-\rho_{0}(t)\right\|_{L^{s}\left(\mathbb{T}^{d}\right)} \leq \begin{cases}M \eta\left\|R_{0}(t)\right\|_{L^{1}\left(\mathbb{T}^{d}\right)}^{1 / s}, & t \in I_{\sigma / 2}, \\
0, & t \in[0,1] \backslash I_{\sigma / 2},\end{cases} \\
& \left\|u_{1}(t)-u_{0}(t)\right\|_{L^{s^{\prime}}\left(\mathbb{T}^{d}\right)} \leq \begin{cases}M \eta^{-1}\left\|R_{0}(t)\right\|_{L^{1}\left(\mathbb{T}^{d}\right)}^{1 / s^{\prime}}, & t \in I_{\sigma / 2}, \\
0, & t \in[0,1] \backslash I_{\sigma / 2},\end{cases} \\
& \left.\begin{array}{l}
\left\|\rho_{1}(t)-\rho_{0}(t)\right\|_{W^{m, p}\left(\mathbb{T}^{d}\right)} \\
\left\|u_{1}(t)-u_{0}(t)\right\|_{W^{\tilde{m}, \tilde{p}}\left(\mathbb{T}^{d}\right)}
\end{array}\right\} \leq \delta \\
& \left\|R_{1}(t)\right\|_{L^{1}\left(\mathbb{T}^{d}\right)} \leq \begin{cases}\delta, & t \in I_{\sigma}, \\
\left\|R_{0}(t)\right\|_{L^{1}\left(\mathbb{T}^{d}\right)}+\delta, & t \in I_{\sigma / 2} \backslash I_{\sigma}, \\
\left\|R_{0}(t)\right\|_{L^{1}\left(\mathbb{T}^{d}\right)}, & t \in[0,1] \backslash I_{\sigma / 2} .\end{cases}
\end{aligned}
$$

Theorem 1.6 can be deduced from Proposition 7.2 exactly in the same way as Theorem 1.2 was deduced from Proposition 3.1. The only difference here is the following. In general, it is not true that $\rho(t) \in L^{p}\left(\mathbb{T}^{d}\right), u(t) \in L^{p^{\prime}}\left(\mathbb{T}^{d}\right)$. Therefore the fact that $\rho u \in C\left((0, T) ; L^{1}\left(\mathbb{T}^{d}\right)\right)$ is proven by showing that $\rho \in C\left((0, T) ; L^{s}\left(\mathbb{T}^{d}\right)\right)$ (thanks to (52a)) and $u \in C\left((0, T) ; L^{s^{\prime}}\left(\mathbb{T}^{d}\right)\right)$ (thanks to (52b)).

Sketch of the proof of Proposition 7.2 The proof is analog to the proof of Proposition 3.1 presented in Sects. 4-6. Here, however, we need modify the "rate of concentration" of the Mikado fields defined in (39) to achieve better estimates on the derivatives. In other words, we have to modify the choice of $a, b$ in (39), as follows. In order to get estimates (52a)-(52b), we want

$$
\left\|\Theta_{\mu}^{j}\right\|_{L^{s}\left(\mathbb{T}^{d}\right)},\left\|W_{\mu}^{j}\right\|_{L^{s^{\prime}}\left(\mathbb{T}^{d}\right)}, \leq \text { const. }
$$

to get $(52 \mathrm{c})$ we want

$$
\left\|\Theta_{\mu}^{j}\right\|_{W^{m, p}} \leq \mathrm{const} \cdot \mu^{-\gamma},\left\|W_{\mu}^{j}\right\|_{W^{\tilde{m}, \tilde{p}}} \leq \mathrm{const} \cdot \mu^{-\gamma}
$$

we also require

$$
\left\|\Theta_{\mu}^{j}\right\|_{L^{1}\left(\mathbb{T}^{d}\right)}, \leq \mathrm{const} \cdot \mu^{-\gamma},\left\|W_{\mu}^{j}\right\|_{L^{1}\left(\mathbb{T}^{d}\right)} \leq \mathrm{const} \cdot \mu^{-\gamma},
$$


for some positive constant $\gamma>0$. Compare (53) with the first and the second estimates in (37), compare (54) with the last estimate in (38) and compare (55) with the first and the second estimates in (38).

We want to find

$$
a, b \in(0, d-1)
$$

so that $a+b=d-1$ and (54) is achieved. If we can do that, then condition (55) is a consequence of (35) and (56). Similarly, condition (53) is automatically satisfied, choosing

$$
s=\frac{d-1}{a}, \quad s^{\prime}=\frac{d-1}{b} .
$$

and observing that (56) implies $s, s^{\prime} \in(1, \infty)$.

Using (35), we see that, to achieve (54), we need

$$
\left\{\begin{array}{l}
a+m-\frac{d-1}{p}<0, \\
b+\tilde{m}-\frac{d-1}{\tilde{p}}<0 .
\end{array}\right.
$$

Notice that, since $a+b=d-1,(57 \mathrm{~b})$ is equivalent to

$$
a>(d-1)\left(1-\frac{1}{\tilde{p}}\right)+\tilde{m} .
$$

It is then possible to find $a, b$ satisfying (56) and (57), with $a+d=d-1$, if and only if

$$
\max \left\{0,(d-1)\left(1-\frac{1}{\tilde{p}}\right)+\tilde{m}\right\}<\min \left\{d-1, \frac{d-1}{p}-m\right\}
$$

and this last condition is equivalent to (14).

Proposition 7.2 can now be proven exactly as we proved Proposition 3.1 in Sects. 46 , this time using (53)-(55) instead of (37)-(38).

\subsection{Sketch of the proof of Theorem 1.10}

Once again, also for Theorem 1.10 there is a main proposition, from which the proof of the theorem follows. Let us consider the equation

$$
\left\{\begin{array}{l}
\partial_{t} \rho+\operatorname{div}(\rho u)-L \rho=-\operatorname{div} R \\
\operatorname{div} u=0
\end{array}\right.
$$

Recall that $L$ is a constant coefficient differential operator of order $k \in \mathbb{N}, k \geq 2$.

Proposition 7.3 Proposition 7.2 holds with (59) instead of (25). 
Sketch of the proof of Proposition 7.3 Similarly to the proof of Proposition 7.2, we want to choose the exponents $a, b \in(0, d-1)$ in Proposition 4.1 so that (53)-(55) are satisfied and, moreover,

$$
\left\|D^{k-1} \Theta_{\mu}^{j}\right\|_{L^{1}\left(\mathbb{T}^{d}\right)} \leq \mathrm{const} \cdot \mu^{-\gamma}
$$

for some positive constant $\gamma>0$. As in Proposition 7.2, to get (53)-(55) we need (58). Moreover, condition (60) is satisfied, provided

$$
a+(k-1)-(d-1)<0,
$$

or, equivalently,

$$
a<d-k \text {. }
$$

Putting together (58) and (61), we obtain the condition

$$
\max \left\{0,(d-1)\left(1-\frac{1}{\tilde{p}}\right)+\tilde{m}\right\}<\min \left\{d-1, d-k, \frac{d-1}{p}-m\right\} .
$$

It is now not difficult to see that the last inequality is satisfied if and only if (17) holds.

Then the perturbations $\vartheta, w, \vartheta_{c}, w_{c}$ can be defined as in Sect. 4.2 and the estimates on the perturbations can be proven as in Proposition 7.2. In the definition of the new defect field we want to define $R_{1}$ so that

$$
-\operatorname{div} R_{1}=\partial_{t} \rho_{1}+\operatorname{div}\left(\rho_{1} u_{1}\right)-L \rho_{1} .
$$

We can write $L=\operatorname{div} \tilde{L}$, where $\tilde{L}$ is a constant coefficient differential operator of order $k-1$. This leads to an additional term $\tilde{L} \vartheta$ in the expression for $R^{\text {linear }}$ (compare with (45)), which can be estimated using (60):

$$
\|\tilde{L} \vartheta\|_{L^{1}\left(\mathbb{T}^{d}\right)} \leq C\left(\eta, \delta,\left\|R_{0}\right\|_{C^{1}}\right) \lambda^{k-1} \mu^{-\gamma}
$$

Choosing $\mu=\lambda^{c}$ with $c>(k-1) / \gamma$, we get the estimates for $\left\|R_{1}(t)\right\|_{L^{1}}$. This concludes the proof of the proposition (and thence also the proof of Theorem 1.10).

Acknowledgements The authors would like to thank Gianluca Crippa for several very useful comments. This research was supported by the ERC Grant Agreement No. 724298.

Open Access This article is distributed under the terms of the Creative Commons Attribution 4.0 International License (http://creativecommons.org/licenses/by/4.0/), which permits unrestricted use, distribution, and reproduction in any medium, provided you give appropriate credit to the original author(s) and the source, provide a link to the Creative Commons license, and indicate if changes were made.

\section{References}

1. Aizenman, M.: On vector fields as generators of flows: a counterexample to Nelson's conjecture. Ann. Math. 107(2), 287-296 (1978) 
2. Alberti, G., Bianchini, S., Crippa, G.: A uniqueness result for the continuity equation in two dimensions. J. Eur. Math. Soc. 16(2), 201-234 (2014)

3. Alberti, G., Crippa, G., Mazzucato, A.L.: Exponential self-similar mixing and loss of regularity for continuity equations. C. R. Math. Acad. Sci. Paris 352(11), 901-906 (2014)

4. Alberti, G., Crippa, G., Mazzucato, A. L.: Exponential self-similar mixing by incompressible flows. arXiv:1605.02090 (2016)

5. Ambrosio, L.: Transport equation and Cauchy problem for BV vector fields. Invent. Math. 158(2), 227-260 (2004)

6. Ambrosio, L.: Well posedness of ODEs and continuity equations with nonsmooth vector fields, and applications. Rev. Mat. Comput. 30(3), 427-450 (2017)

7. Ambrosio, L., Colombo, M., Figalli, A.: Existence and uniqueness of maximal regular flows for nonsmooth vector fields. Arch. Rational Mech. Anal. 218, 1043-1081 (2015)

8. Bianchini, S., Bonicatto, P. A uniqueness result for the decomposition of vector fields in Rd. SISSA (2017)

9. Bianchini, S., Colombo, M., Crippa, G., Spinolo, L.V.: Optimality of integrability estimates for advection-diffusion equations. Nonlinear Differ. Equ. Appl. NoDEA 24(4), 33 (2017)

10. Buckmaster, T.: Onsager's conjecture almost everywhere in time. Commun. Math. Phys. 333(3), 11751198 (2015)

11. Buckmaster, T., De Lellis, C., Isett, P., Székelyhidi Jr., L.: Anomalous dissipation for 1/5-Hölder Euler flows. Ann. Math. 182(1), 127-172 (2015)

12. Buckmaster, T., De Lellis, C., Székelyhidi Jr., L.: Dissipative Euler flows with Onsager-critical spatial regularity. Commun. Pure Appl. Math. 9, 1613-1670 (2016)

13. Buckmaster, T., De Lellis, C., Székelyhidi Jr, L., Vicol, V.: Onsager's conjecture for admissible weak solutions. arXiv:1701.08678 (2017)

14. Buckmaster, T., Vicol, V.: Nonuniqueness of weak solutions to the Navier-Stokes equation. arXiv:1709.10033 (2017)

15. Caravenna, L., Crippa, G.: Uniqueness and Lagrangianity for solutions with lack of integrability of the continuity equation. C. R. Math. Acad. Sci. Paris 354(12), 1168-1173 (2016)

16. Colombini, F., Lerner, N.: Uniqueness of continuous solutions for BV vector fields. Duke Math. J. 111(2), 357-384 (2002)

17. Colombini, F., Luo, T., Rauch, J.: Uniqueness and nonuniqueness for nonsmooth divergence free transport. In: Seminaire: Équations aux Dérivées Partielles, 2002-2003. École Polytech., Palaiseau, 2003, pp. Exp. No. XXII-21

18. Crippa, G., Gusev, N., Spirito, S., Wiedemann, E.: Non-uniqueness and prescribed energy for the continuity equation. Comm. Math. Sci. 13(7), 1937-1947 (2015)

19. Crippa, G., Spirito, S.: Renormalized solutions of the 2D Euler equations. Commun. Math. Phys. 339(1), 191-198 (2015)

20. Daneri, S., Székelyhidi Jr., L.: Non-uniqueness and h-principle for Hölder-continuous weak solutions of the Euler equations. Arch. Rational Mech. Anal. 224(2), 471-514 (2017)

21. De Lellis, C., Székelyhidi Jr., L.: The Euler equations as a differential inclusion. Ann. Math. 170(3), 1417-1436 (2009)

22. De Lellis, C., Székelyhidi Jr., L.: Dissipative continuous Euler flows. Invent. Math. 193(2), 377-407 (2012)

23. De Lellis, C., Székelyhidi Jr., L.: Dissipative Euler flows and Onsager's conjecture. J. Eur. Math. Soc. 16(7), 1467-1505 (2014)

24. Depauw, N.: Non unicité des solutions bornées pour un champ de vecteurs BV en dehors d'un hyperplan. C. R. Math. Acad. Sci. Paris 337(4), 249-252 (2003)

25. DiPerna, R.J., Lions, P.-L.: On the Cauchy problem for Boltzmann equations: global existence and weak stability. Ann. Math. 130(2), 321-366 (1989)

26. DiPerna, R.J., Lions, P.-L.: Ordinary differential equations, transport theory and Sobolev spaces. Invent. Math. 98(3), 511-547 (1989)

27. Frisch, U.: Turbulence. Cambridge University Press, Cambridge (1995)

28. Frisch, U., Sulem, P.-L., Nelkin, M.: A simple dynamical model of intermittent fully developed turbulence. J. Fluid Mech. 87(04), 719-736 (1978)

29. Isett, P.: A Proof of Onsager's Conjecture. Ann. Math. 188(3), 871-963 (2016)

30. Isett, P., Vicol, V.: Hölder continuous solutions of active scalar equations. Ann. PDE 1(1), 2-77 (2015) 
31. Ladyženskaja, O. A., Solonnikov, V., Ural'ceva, N.: Linear and quasilinear equations of parabolic type. Vol. 23, Series in Translations of Mathematical Monographs. American Mathematical Society, 648pp (1968)

32. Le Bris, C., Lions, P.-L.: Renormalized solutions of some transport equations with partially $W^{1,1}$ velocities and applications. Annali di Matematica Pura ed Applicata 183(1), 97-130 (2004)

33. Le Bris, C., Lions, P.-L.: Existence and uniqueness of solutions to Fokker-Planck type equations with irregular coefficients. Commun. Partial Differ. Equ. 33(7), 1272-1317 (2008)

34. Lévy, G.: On uniqueness for a rough transport-diffusion equation. C. R. Math. Acad. Sci. Paris 354(8), 804-807 (2016)

35. Lions, P.-L.: Mathematical Topics in Fluid Mechanics: Volume 1: Incompressible Models. Oxford University Press, Oxford (1996)

36. Lions, P.-L.: Mathematical topics in fluid mechanics. Vol. 2, vol. 10 of Oxford Lecture Series in Mathematics and Its Applications. The Clarendon Press, New York (1998)

37. Yao, Y., Zlato, A.: Mixing and un-mixing by incompressible flows. J. Eur. Math. Soc. 19(7), 1911-1948 (2017) 